\title{
Constraining the properties of AGN host galaxies with spectral energy distribution modelling
}

\author{
L. Ciesla ${ }^{1,2}$, V. Charmandaris ${ }^{2,1,3}$, A. Georgakakis ${ }^{4,2}$, E. Bernhard ${ }^{5}$, P. D. Mitchell ${ }^{6}$, V. Buat ${ }^{7}$, D. Elbaz ${ }^{8}$, E. LeFloc'h ${ }^{8}$, \\ C. G. Lacey ${ }^{6}$, G. E. Magdis ${ }^{9,2}$, and M. Xilouris ${ }^{2}$
}

1 University of Crete, Department of Physics, 71003 Heraklion, Greece e-mail: ciesla@physics.uoc.gr

2 Institute for Astronomy, Astrophysics, Space Applications and Remote Sensing, National Observatory of Athens, 15236 Penteli, Greece

${ }^{3}$ Chercheur Associé, Observatoire de Paris, 75014 Paris, France

${ }^{4}$ Department of Physics and Astronomy, Max Planck Institut für Extraterrestrische Physik, Giessenbachstraße, 85748 Garching, Germany

5 Department of Physics \& Astronomy, University of Sheffield, Sheffield S3 7RH, UK

${ }^{6}$ Institute for Computational Cosmology, Department of Physics, University of Durham, South Road, Durham DH1 3LE, UK

7 Aix-Marseille Université, CNRS, LAM (Laboratoire d'Astrophysique de Marseille) UMR7326, 13388 Marseille, France

${ }^{8}$ Laboratoire AIM, CEA/DSM/IRFU, CNRS, Université Paris-Diderot, 91190 Gif, France

9 Department of Physics, University of Oxford, Keble Road, Oxford OX1 3RH, UK

Received 31 October 2014 / Accepted 15 January 2015

\begin{abstract}
Detailed studies of the spectral energy distribution (SED) of normal galaxies have increasingly been used to understand the physical mechanism dominating their integrated emission, mainly owing to the availability of high quality multi-wavelength data from the UV to the far-infrared (FIR). However, systems hosting dust-enshrouded nuclear starbursts and/or an accreting supermassive black hole (an active galactic nucleus or AGN) are especially challenging to study. This is due to the complex interplay between the heating by massive stars and the AGN, the absorption and emission of radiation from dust, as well as the presence of the underlying old stellar population. We used the latest release of CIGALE, a fast state-of-the-art galaxy SED-fitting model relying on energy balance, to study the influence of an AGN in a self consistent manner in estimating both the star formation rate (SFR) and stellar mass in galaxies, as well as to calculate the contribution of the AGN to the power output of the host. Using the semi-analytical galaxy formation model GALFORM, we created a suite of mock galaxy SEDs using realistic star formation histories (SFH). We also added an AGN of Type-1, Type-2, or intermediate-type whose contribution to the bolometric luminosity can be variable. We performed an SED-fitting of these catalogues with CIGALE, assuming three different SFHs: a single-exponentially-decreasing ( $1 \tau$-dec), a double-exponentiallydecreasing $(2 \tau-\mathrm{dec})$, and a delayed SFH. Constraining the overall contribution of an AGN to the total infrared luminosity ( frac $_{\mathrm{AGN}}$ ) is very challenging for $\mathrm{frac}_{\mathrm{AGN}}<20 \%$, with uncertainties of $\sim 5-30 \%$ for higher fractions depending on the AGN type, while FIR and sub-mm are essential. The AGN power has an impact on the estimation of $M_{*}$ in Type- 1 and intermediate-type AGNs but has no effect on galaxies hosting Type-2 AGNs. We find that in the absence of AGN emission, the best estimates of $M_{*}$ are obtained using the $2 \tau$-dec model but at the expense of realistic ages of the stellar population. The delayed SFH model provides good estimates of $M_{*}$ and SFR, with a maximum offset of $10 \%$ as well as better estimates of the age. Our analysis shows that the under-estimation of the SFR increases with frac $_{\mathrm{AGN}}$ for Type-1 systems, as well as for low contributions of an intermediate AGN type, but it is quite insensitive to the emission of Type-2 AGNs up to $\mathrm{frac}_{\mathrm{AGN}} \sim 45 \%$. A lack of sampling the FIR, or sub-mm domain systematically over-estimates the SFR $(<20 \%)$, independent of the contribution of the AGN. Similarly, the UV emission is critical in accurately retrieving both the $M_{*}$ for Type-1 and intermediate- type AGN and the SFR of all three AGN types. We show that the presence of AGN emission introduces a scatter to the SFR- $M_{*}$ main sequence relation derived from SED-fitting, which is driven by the uncertainties on $M_{*}$. Finally, we used our mock catalogues to test the popular IR SED-fitting code DECOMPIR and show that rrac $_{\mathrm{AGN}}$ is under-estimated but that the SFR is recovered well for Type-1 and intermediate-types of AGN. The frac ${ }_{\mathrm{AGN}}$, SFR, and $L_{\mathrm{IR}}$ estimates of Type-2 AGNs are more problematic owing to a FIR emission disagreement between predicted and observed models.
\end{abstract}

Key words. galaxies: fundamental parameters - galaxies: active

\section{Introduction}

The formation of galaxies and their evolution with cosmic time are open problems in current astrophysical research. Understanding the assembly of galaxies and the build-up of their stellar populations is challenging because the relevant physical processes are complex and interconnected, and they operate on a wide range of scales. Gas inflows from the intergalactic medium (IGM) for example, are important for supplying galaxies with fresh material that can be turned into stars or feed supermassive black holes (SMBH) at their centres. Feedback processes associated with stellar evolution of active galactic nuclei (AGN) can drastically modify the physical conditions of the Inter-Stellar Medium (ISM), thereby affecting the formation of new stars. Supernovae explosions enrich the gaseous component of galaxies and their environments with heavy metals and therefore change the composition of the ISM with implications for star formation. The density of IGM on large scales or 
interactions with nearby galaxies also have an impact on the ISM of individual systems and can modify their evolutionary path.

One approach for shedding light on the physics of galaxy formation and evolution is population studies. Multi-wavelength observations provide information on galaxy properties such as stellar mass, star formation history (SFH), gas content, AGN activity, kinematics, structural parameters or position on the cosmic web. Each of these observationally determined parameters probe different physical processes. Exploring correlations between them for large samples can therefore provide insight onto how galaxies form and evolve in the Universe. For example, the discovery of the bimodality of galaxies on the colour (proxy to SFH) versus stellar-mass plane (e.g. Strateva et al. 2001; Baldry et al. 2004, 2006; Bell et al. 2004) has been interpreted as evidence of star formation quenching that may be driven by either internal (e.g. feedback) or external (e.g. environment) processes (e.g. Bell et al. 2004; Faber et al. 2007; Schawinski et al. 2014). The star formation main sequence (MS), i.e. the relatively tight correlation between stellar mass and star formation rate (SFR) of star-forming galaxies (e.g. Salim et al. 2007; Noeske et al. 2007b; Elbaz et al. 2007; Rodighiero et al. 2011; Speagle et al. 2014) suggests that the bulk of the stars in the Universe form via secular processes rather than in violent events, such as mergers. Additionally, the slope and redshift evolution of the MS normalization have been discussed in the context of feedback processes and gas exhaustion with cosmic time, respectively (e.g. Noeske et al. 2007a; Zheng et al. 2007; Tacconi et al. 2013; Guo et al. 2013). Correlations between galaxy structural parameters, SFR and stellar mass (e.g. Kauffmann et al. 2004, Wuyts et al. 2011, Bell et al. 2012, Lang et al. 2014) suggest that the formation of galaxy bulges and the quenching of star formation are likely related to the same underlying processes.

Amongst the different galaxy properties that are accessible to observations, the stellar mass and SFR play an important role in galaxy evolution studies. This is not surprising. Both quantities provide a measure of the SFH of the galaxy, either integrated over its lifetime (stellar mass) or averaged in the past few tens to a few hundred million years (instantaneous SFR). Indeed, all galaxy properties show strong correlations with either stellar mass, SFR or both (e.g., Kauffmann et al. 2004). This has led to an increasing refinement in methodology to provide more accurate and less biased observational constraints to the stellar mass and SFR of individual galaxies. Among the different approaches, the one that has been extensively used in the literature is the use of stellar population synthesis models (e.g. Bruzual \& Charlot 2003; Maraston 2005) to generate spectral energy distribution (SED) templates for different SFH and then fit them to the broad-band photometry of galaxies (e.g. Walcher et al. 2011, and references therein). This is driven by the explosion in recent years in the quality and quantity of multi-wavelength imaging surveys, which provide UV to infrared (IR) SEDs of large galaxy samples over a wide range of redshifts. This approach for inferring galaxy parameters has also been extensively tested to identify limitations and potential sources of systematics (e.g. Pozzetti et al. 2007; Marchesini et al. 2009; Conroy et al. 2009; Wuyts et al. 2009; Ilbert et al. 2010; Michałowski et al. 2012; Pforr et al. 2012; Banerji et al. 2013; Schaerer et al. 2013; Mitchell et al. 2013; Buat et al. 2014).

The overall consensus is that stellar masses can be reliably constrained, although systematics up to the 0.5 dex level remain, depending on the adopted initial mass function, stellar population libraries, functional form of the model SFH, and the implementation of dust attenuation. The SFR of galaxies is often determined using the observed luminosity at specific wavelengths as tracers (e.g. Kennicutt 1998; Hopkins 2004). Nevertheless, studies that fit the full SED to derive SFR find overall reasonable agreement with estimates based on specific tracers (e.g. Salim et al. 2007, 2009; Walcher et al. 2008).

One aspect of galaxy evolution that has developed considerably in recent years is the relation to SMBH growth. This has been motivated by the tight correlations between proxies of the stellar mass of local spheroids and the mass of the black hole at their centres (e.g. Kormendy \& Ho 2013, and references therein). One interpretation of these correlations is that $\mathrm{AGN}$ and galaxies co-evolve as dictated by a common gas reservoir that forms new stars and also feeds the central black hole. An alternative explanation is that AGN outflows heat and/or expel the cold gas component of galaxies, thereby regulating the formation of new stars and ultimately the accretion onto the central SMBH itself (e.g. Silk \& Rees 1998; Fabian 1999; King 2003, 2005; Di Matteo et al. 2005). There is indeed increasing observational evidence for AGN-driven winds in the local Universe and at high redshift (e.g. Crenshaw et al. 2003; Blustin et al. 2005; Tombesi et al. 2010, 2012; Saez \& Chartas 2011; Lanzuisi et al. 2012; Cicone et al. 2012, 2014; Harrison et al. 2014). What remains controversial, however, is how common these outflows are and whether they are energetic enough to affect the ISM of their hosts. One way to approach these issues and place AGNs in the context of galaxy evolution is statistical studies of the host galaxy properties of large AGN samples. Questions that this approach could address include, how black hole growth is related to star formation, when accretion onto the central SMBH is triggered during the lifetime of a galaxy and whether AGNs affect their host galaxy properties.

Multi-wavelength survey programmes in the past decade have started addressing these questions. Far-infrared (FIR) and sub-mm surveys with Herschel for example, measure the mean SFR of AGN hosts via stacking methods and show that AGN lie, on average, on the star formation main sequence at all redshifts (e.g. Mullaney et al. 2012; Santini et al. 2012; Rosario et al. 2012, 2013). This finding suggests that at least in an average sense the same physical processes govern the formation of stars in galaxies and the growth of black holes in their centres (Silverman et al. 2009; Georgakakis et al. 2011; Mullaney et al. 2012). Studies of the star formation properties of individual AGN hosts (rather than averages over populations) suggest a wide range of SFH. The rest-frame colours of AGN hosts (Aird et al. 2012; Georgakakis et al. 2014; Azadi et al. 2014) or their position on the colour-magnitude diagram (e.g. Sánchez et al. 2004; Nandra et al. 2007; Schawinski et al. 2009) indicate that they are scattered at the red sequence of passive galaxies, the star-forming cloud and the green valley in between. A higher incidence of AGN among green valley galaxies is claimed (Nandra et al. 2007; Schawinski et al. 2009), i.e. systems with colours in the transition region between the red sequence and the blue cloud. This can be interpreted that AGN are responsible for quenching star formation in galaxies, hence their transition from blue star-forming to red and dead systems. At the same time however, the importance of constructing appropriate control samples when determining the fraction of AGN among galaxies is also emphasized. The evidence of an increased AGN fraction amongst green valley galaxies is less strong, or may even disappear once the stellar mass of AGN hosts and the control galaxy samples are matched (e.g. Silverman et al. 2009; Xue et al. 2010; Aird et al. 2012). Constraints on the stellar ages of AGN hosts further suggests that black hole accretion primarily occurs a few hundred to a few thousand million years after the 
peak of star formation (Kauffmann et al. 2003a; Wild et al. 2010; Hernán-Caballero et al. 2014), i.e. during a period when galaxies can be identified as post-starbursts (e.g. Goto 2006; Georgakakis et al. 2008). A time lag between the peak of star formation and the black hole growth may pose a problem to AGN-driven star formation quenching scenarios.

The studies above demonstrate the importance of measuring the star formation and stellar mass for AGN hosts. This is not only for understanding the relation between SFH and black hole growth in individual systems, but also for constructing appropriate control samples of inactive (non-AGN) galaxies. Determining stellar masses or SFR for AGN hosts is challenging. Emission from the central engine contaminates or may even dominate the underlying galaxy light, thereby rendering the determination of galaxy properties, such as by SED-fitting methods, difficult. Different approaches have been adopted in the literature to address this issue. Standard SED-fitting methods are often applied to determine host galaxy properties only for low-luminosity and obscured AGN, under the reasonable assumption that contamination in these objects is low. A potential problem with this approach is that under certain models for the co-evolution of AGN and galaxies (e.g. Hopkins et al. 2006), different levels of obscuration and different accretion luminosities correspond to different evolutionary phases in the black hole growth. The selection against unobscured and luminous AGN might introduce biases and lead to erroneous conclusions on the relation between black hole accretion and galaxy formation. An alternative approach is to add AGN templates to the stellar template library and perform SED deconvolution to separate the galaxy and AGN components (e.g. Bongiorno et al. 2012; Lusso et al. 2011, 2013; Rovilos et al. 2014). This can be powerful, although degenerations between AGN and galaxy templates may introduce systematics in the determination of host galaxy parameters.

To date, there are still a few studies that explore and quantify the impact of AGN contamination on the determination of the underlying host galaxy properties via SED-fitting (Wuyts et al. 2011; Hayward \& Smith 2015). In this paper, we address this using the Bayesian-based SED-fitting code CIGALE (Noll et al. 2009, Burgarella et al., in prep.; Boquien et al., in prep.). To do so, we follow the method developed in Mitchell et al. (2013) and use simulated galaxies from the SAM (Semi-Analytica Model) code GALFORM from which we know the exact value of the stellar mass and SFR. After building the UV-to-sub-mm SEDs of the GALFORM objects and adding an AGN contribution, we study our ability to retrieve the original properties using CIGALE.

The paper is organized as follows. First, we introduce GALFORM and present the derived SFH of a hundred galaxies at $z=1$ (Sect. 2). We describe how we build our mock SEDs using the modelling function of CIGALE in Sect. 2, and how we perform the SED fitting in Sect. 3. In the same section, we also present the comparison between the true values of the stellar masses and SFRs and the outputs of CIGALE in normal galaxies as well as in AGN host galaxies. Finally, we discuss the impact of our results on the SFR- $M_{*}$ relation in Sect. 4. In Sect. 5, we use our mock samples to evaluate the performance of the popular IR SED-fitting code DECOMPIR (Mullaney et al. 2011). The purpose of this work is not to test the validity of AGN emission models thoroughly or the ability of SED-fitting codes to accurately retrieve the properties of the AGN through its IR emission but instead to analyse the impact of this emission on the derivation of the basic host galaxy properties.

In this work, we assume that $\Omega_{\mathrm{m}}=0.25, \Omega_{\Lambda}=0.75$, and $H_{0}=73 \mathrm{~km} \mathrm{~s}^{-1} \mathrm{Mpc}^{-1}$. These values are used because the
GALFORM model is built on top of merger trees generated from the original Millenium simulation (Springel et al. 2005), which assumes a WMAP1-like cosmology. This choice does not affect our results, because we only use this cosmology to convert physical quantities to observables without comparisons with other works based on more recent cosmological parameters. All of the stellar masses and SFRs are provided assuming an initial mass function (IMF) of Salpeter.

\section{Building a realistic mock galaxy sample}

In this paper we explore the problem of AGN and stellar light decomposition via SED template fits to multi-wavelength broadband photometric data. The goal is to quantify how well the galaxy properties of AGN hosts, such as stellar mass and star formation rate, can be measured from broad-band photometry. Our approach is to use mock galaxies extracted from the GALFORM SAM (Cole et al. 2000), for which the SFHs, stellar masses $\left(M_{*}\right)$ and instantaneous SFR are known. This information allows the construction of UV to sub-mm SEDs for the mock galaxies. AGN templates are then added to the galaxy emission to generate composite SEDs. These are then integrated within broadband filters to generate mock photometric catalogues. The fitting modules of the CIGALE code are then applied to the mock galaxy photometry to decompose AGN from stellar light. Each of these steps is described in the following sections.

\subsection{Simulations of realistic star formation histories}

To generate our grid of mock SFHs, we used the GALFORM semianalytic galaxy formation model to simulate the assembly of the galaxy population within the context of the $\Lambda \mathrm{CDM}$ model of structure formation (Cole et al. 2000; Bower et al. 2006; Benson $\&$ Bower 2010). The model was constructed on top of dark matter halo merger trees extracted from the Millennium dark matter $N$-body simulation (Springel et al. 2005). Within each halo, the baryonic mass is divided into hot and cold gas, as well as into stellar disk and bulge components. The model then solves a set of coupled differential equations that describe how mass is exchanged between these different components. Star formation in the model is split into quiescent star formation that occurs in galaxy disks and bursts of star formation that are triggered by galaxy mergers and disk instabilities (Baugh et al. 2005; Bower et al. 2006; Lagos et al. 2011).

Star formation histories are extracted from the fiducial version of the model presented by Mitchell et al. (2014). We randomly select a total of 100 galaxies from that simulation at redshift $z=1$. Motivated by observational evidence that AGN hosts are typically massive and lie, at least in average sense, on the main sequence of star formation (e.g., Mullaney et al. 2012; Santini et al. 2012; Rosario et al. 2012, 2013), we also choose the mock galaxies to sit on the main sequence of the SAM at redshift $z=1$ and to have high stellar masses. In particular, they fulfil the following criteria:

1. Specific star-formation rates $\mathrm{S} S F R>0.1 \mathrm{Gyr}^{-1}$. This cut separates main star formation sequence from passive galaxies in the model at $z=1$ (Mitchell et al. 2014).

2. Stellar mass in the range $10<\log \left(M_{*} / M_{\odot}\right)<11$.

For these SFHs, we sum over the star formation in all progenitors of the final galaxy and we also combine the stellar mass assembly of the disk and bulge together. Bursts of star formation in the model can occur over relatively short timescales in some cases, 


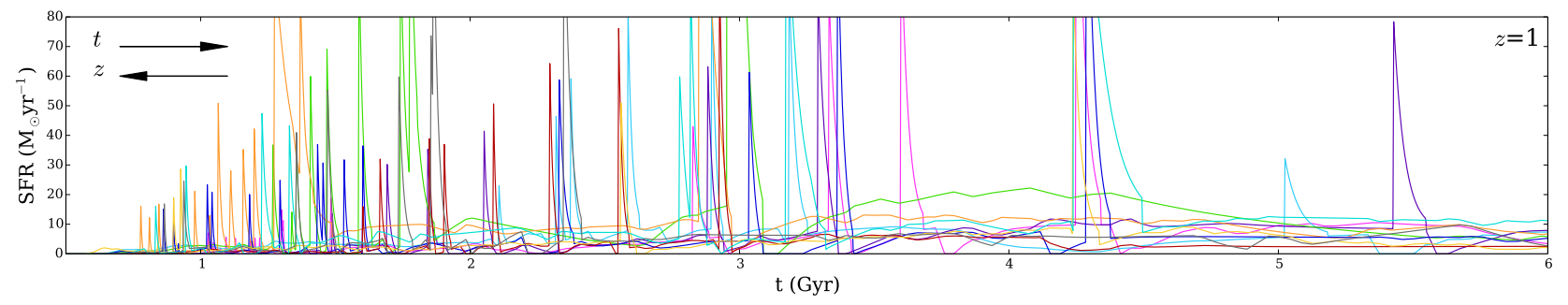

Fig. 1. Examples of $10 \mathrm{SFHs}$ of simulated galaxies extracted from the GALFORM SAM.
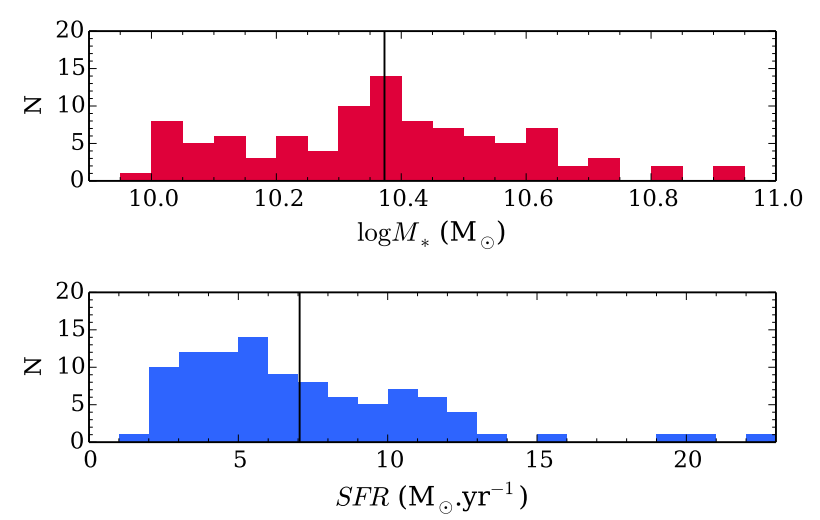

Fig. 2. Distribution of $M_{*}$ and SFR associated with the GALFORM SFHs. The black lines show the mean value of the distributions.

so we construct SFHs from the model to have high temporal resolution. We show ten examples of the produced SFHs in Fig. 1. The distribution of stellar mass and SFR at redshft $z=1$ of the 100 simulated galaxies selected for our analysis are presented in Fig. 2. The mean values of the sample are $\log M_{*}=10.37 M_{\odot}$ and $S F R=7.05 M_{*} \mathrm{yr}^{-1}$.

\subsection{Simulations of UV to sub-mm SEDs}

CIGALE $^{1}$ is a package that has two different and independent functions: an SED modelling function (Boquien et al., in prep.) and an SED-fitting function (Burgarella et al., in prep.). The baseline functions of CIGALE are presented in Noll et al. (2009). Based on the same general principles as the original version of CIGALE, this entirely new version has been designed for a broader set of scientific applications and better performance. The latest version of the modelling function of CIGALE is briefly described in this section.

The SED modelling function of CIGALE allows construction of galaxy SEDs from the UV to the sub-mm by assuming a stellar population library and SFHs provided by the user. CIGALE builds the SED taking the energy balance into account, i.e., the energy absorbed by dust in UV-optical is re-emitted in the IR.

In CIGALE, the SFH can be handled in two different ways. The first, is to model it using simple analytic functions (e.g. exponential forms and delayed SFHs). The second is to provide more complex (non-analytic) SFHs (e.g., Boquien et al. 2014), such as those provided by the GALFORM SAM. The stellar population models of either Maraston (2005) or Bruzual \& Charlot (2003) are convolved with the adopted SFH to produce stellar SEDs, which are then attenuated by dust. The energy absorbed by the dust is re-emitted in the IR using a choice of different

\footnotetext{
The code is freely available at: http://cigale. lam.fr/
}

dust templates (Dale \& Helou 2002; Dale et al. 2014; Draine \& Li 2007; Casey 2012).

CIGALE also allows the emission from AGN to be added to the stellar SED. The AGN templates from the library of Fritz et al. (2006) are adopted. These SEDs consist of two components. The first one is the isotropic emission of the central source, which is assumed to be point-like. This emission is a composition of power laws with variable indices in the wavelength range of $0.001-20 \mu \mathrm{m}$. The second component of the Fritz et al. (2006) models is radiation from dust with a toroidal geometry in the vicinity of the central engine. Part of the direct emission of the AGN is either absorbed by the toroidal obscurer and re-emitted at longer wavelengths $(1-1000 \mu \mathrm{m})$ or scattered by the same medium. Dust can be optically thick to its own radiation, thus requiring the numerical resolution of the radiative transfer problem. In Fritz et al. (2006) models, the conservation of energy is always verified within $1 \%$ for typical solutions, and up to $10 \%$ in the case of very high optical depth and nonconstant dust density. The choice of adding the Fritz et al. (2006) library into CIGALE is driven by the energy balance handling of the two components, which also matches the energy conservation philosophy of CIGALE. Furthermore, this library has been tested in numerous studies of the literature (e.g., Fritz et al. 2006; Hatziminaoglou et al. 2008, 2010; Feltre et al. 2012).

The relative normalization of these AGN components to the host galaxy SED is handled through a parameter that is the fraction of the total IR luminosity due to the AGN so that

$L_{\mathrm{IR}}^{\mathrm{AGN}}=f r a c_{\mathrm{AGN}} \times L_{\mathrm{IR}}^{\mathrm{TOT}}$,

where $L_{\mathrm{IR}}^{\mathrm{AGN}}$ is the AGN IR luminosity, frac $\mathrm{AGN}_{\mathrm{A}}$ is the contribution of the AGN to the total IR luminosity $\left(L_{\mathrm{IR}}^{\mathrm{TOT}}\right)$, i.e. $L_{\mathrm{IR}}^{\mathrm{starburst}}+L_{\mathrm{IR}}^{\mathrm{AGN}}$. Thus, estimating $L_{\mathrm{IR}}^{\mathrm{AGN}}$ depends on the constraints on frac $_{\mathrm{AGN}}$.

For each SFH provided by GALFORM, we compute a host galaxy SED using the Maraston (2005) stellar population models and assuming the Calzetti et al. (2000) extinction law. The amount of reddening, $E(B-V)_{*}$, is chosen to be 0.2 , justified by observational studies of AGN host galaxies (Kauffmann et al. 2003b; Hainline et al. 2012). The energy absorbed in UVOpt-NIR is re-injected in IR, providing the normalization of the Draine \& Li (2007) models in order to maintain the energy balance of the SED. The template is chosen following the results of Magdis et al. (2012) who fit high- $z$ galaxies with the Draine $\&$ Li (2007) models. The parameters used to model the galaxies are presented in Table 1.

We selected three AGN model templates from the Fritz et al. (2006) library to be added to the galaxy SEDs. These include a Type-1 AGN (i.e. unobscured), a Type-2 AGN (i.e. obscured) and a template that lies between the first two and is referred to as intermediate type. The latter model displays a power-law spectral shape in the mid-IR without strong UV/optical emission. Previous studies indicate that such an intermediate template 
Table 1. Galaxy and AGN parameters adopted to generate mock galaxy SEDs.

\begin{tabular}{|c|c|c|}
\hline Parameter & Value & Description \\
\hline \multicolumn{3}{|r|}{ Dust attenuation } \\
\hline$E(B-V)_{*}$ & 0.2 & \\
\hline \multicolumn{3}{|c|}{ Dust template: Draine \& Li (2007) } \\
\hline$q_{\mathrm{PAH}}(\%)$ & 3.19 & Mass fraction of PAH to the total dust mass. \\
\hline$U_{\min }$ & 8.0 & Min. intensity of the interstellar radiation field. \\
\hline$U_{\max }$ & $10^{6}$ & Max. intensity of the interstellar radiation field. \\
\hline$\gamma(\%)$ & 2 & $\begin{array}{l}\text { Relative contribution between dust heated in photodissociation. } \\
\text { regions, and dust heated by diffuse stellar population. }\end{array}$ \\
\hline \multicolumn{3}{|r|}{ AGN emission } \\
\hline$R_{\max } / R_{\min }$ & 60 & Ratio between outer and inner radius of the torus. \\
\hline$\tau_{9.7}$ & $\begin{array}{c}1.0 \text { (for int. type) } \\
6.0 \text { (for Type- } 1 \text { and Type-2) }\end{array}$ & Optical depth at $9.7 \mu \mathrm{m}$. \\
\hline$\beta$ & -0.5 & Linked to the radial dust distribution in the torus. \\
\hline$\gamma$ & 0.0 & Linked to the angular dust distribution in the torus. \\
\hline$\psi$ & $\begin{array}{c}0.001 \text { (for int. type and Type-2) } \\
89.9 \text { (for Type-1) }\end{array}$ & Angle with line of sight. \\
\hline$\theta$ & 100 & Angular opening angle of the torus. \\
\hline frac $_{\mathrm{AGN}}$ & $\begin{array}{l}0 ., 0.05,0.1,0.15,0.2,0.25,0.3 \\
0.35,0.4,0.45,0.5,0.55,0.6,0.7\end{array}$ & Contribution of the AGN to the total $L_{\mathrm{IR}}$. \\
\hline
\end{tabular}

is indeed needed to represent the diversity of the observed SEDs of AGN (Hatziminaoglou et al. 2008, 2009; Feltre et al. 2012). Fritz et al. (2006) model parameters for the three templates are presented in Table $1^{2}$. The parameters for the Type- 1 and Type- 2 AGN templates are representative of local unobscured and obscured AGN, respectively (Fritz et al. 2006). For the intermediate AGN type we adopt one of the Fritz et al. (2006) models with low equatorial optical depth (Hatziminaoglou et al. 2008, 2009; Feltre et al. 2012; Buat et al., in prep.). The three AGN templates adopted in this paper are the minimum required to represent the variety of the broad-band AGN SEDs. There are strong degeneracies among different parameters of the Fritz et al. (2006) library that cannot be broken by multi-wavelength photometric data alone. Additional parameters combinations to those shown in Table 1 do not necessarily result in AGN broad-band SEDs that are distinctively different from our Type 1, Type-2, and intermediate-type models. In Fig. 3, we show the SEDs corresponding to one of the SFHs presented in Fig. 1.

The modelled SEDs are integrated within the broad-band filters of Table 2 to produce mock photometric catalogues. These are used in the next section to assess the reliability of AGN/galaxy decomposition using photometric data and quantify the level of accuracy at which physical parameters of the underlying galaxy can be derived. Random noise is added to the fluxes of each source assuming Gaussian errors with standard deviation $10 \%$ of the flux in a given waveband. We associate a photometric error of $15 \%$ with all the flux densities. The broad-band filters of Table 2 sample a wide range of wavelengths, as shown in Fig. 3. To quantify the level of AGN contribution in each of these filters, we show in Fig. 4 the flux density ratios of the SEDs that include an AGN component relative to those that do not $\left(\operatorname{frac}_{\mathrm{AGN}}=0\right)$. A Type-1 AGN with a $f r a c_{\mathrm{AGN}}=10 \%$ will have $\mathrm{AGN}$ emission that is higher by a factor of 2 in the UV and MIR rest frame compared to the same SED without an AGN component. In the UV

\footnotetext{
2 We note an error in the definition of the angle relative to the line of sight $\psi$ in Fritz et al. (2006): $\psi=0^{\circ}$ corresponds to a Type-2 AGN, whereas an angle $\psi=90^{\circ}$ is for Type- 1 (Fritz, priv. comm.).
}

Table 2. Broad-band filter-set used in this paper.

\begin{tabular}{llc}
\hline \hline Telescope/camera & Filter name & $\lambda_{\text {mean }}(\mu \mathrm{m})$ \\
\hline MOSAIC & $U$ & 0.358 \\
HST & ACS435 & 0.431 \\
& ACS606 & 0.573 \\
& ACS775 & 0.762 \\
Subaru/MOIRCS & ACS850 & 0.9 \\
CFHT/WIRCam & Ks & 1.2 \\
Spitzer & IRAC1 & 2.2 \\
& IRAC2 & 3.6 \\
& IRAC3 & 5.5 \\
& IRAC4 & 8 \\
& IRS16 & 16 \\
& MIPS1 & 24 \\
Herschel & MIPS2 & 70 \\
& PACS green & 100 \\
& PACS red & 160 \\
& PSW & 250 \\
& PMW & 350 \\
& PLW & 500 \\
\hline
\end{tabular}

rest frame, a fraction $\mathrm{frac}_{\mathrm{AGN}}=40 \%$ is sufficient for dominating the emission, with an AGN contribution four times higher than the young stellar population emission. In the MIR rest frame, the AGN emission is dominant for frac $_{\mathrm{AGN}}>20 \%$ with an emission three times higher than the stellar emission. The UV and the MIR are thus key domains for performing AGN/galaxy decomposition in the case of a Type-1 AGN SED as also shown in previous work (e.g., Weedman et al. 2004; Wu et al. 2009). The AGN emission of the intermediate-type is visible in the MIR domains, especially at $4 \mu \mathrm{m}$ rest frame, where it is brighter than the host galaxy by a factor of 2 for a fraction of $10 \%$. For the Type- 2 AGN, it is clear that the emission of the AGN cannot be detected below $2 \mu \mathrm{m}$ rest frame. In this model, the torus is optically thick and the emission from the inner, hotter part of the 

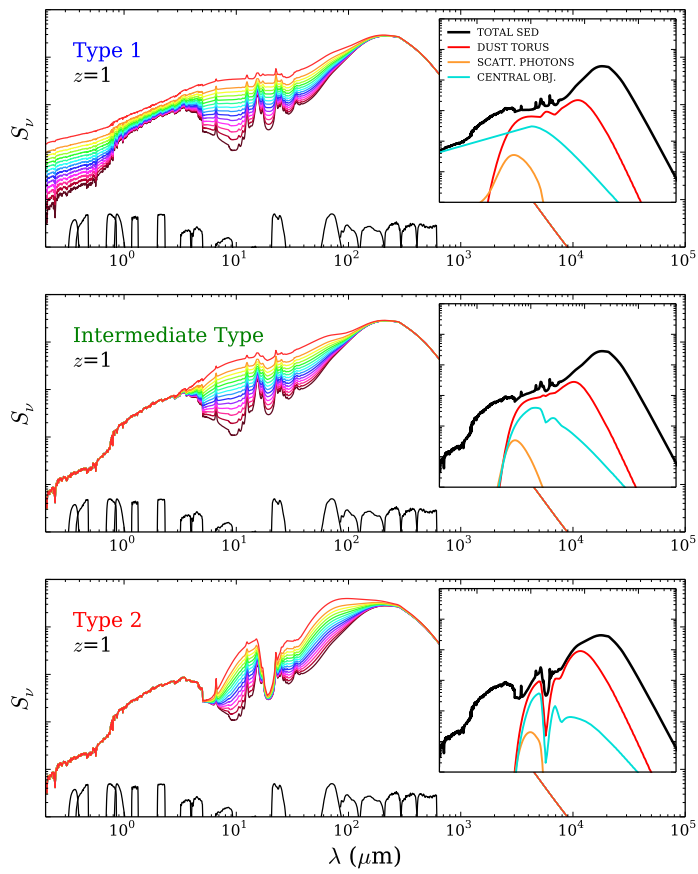

Fig. 3. SEDs generated by GIGALE using a particular SFH extracted from GALFORM and adding on different AGN templates with different normalizations relative to the galaxy light. Each panel corresponds to one of the three Fritz et al. (2006) templates presented in Table 1: the upper panel is for Type-1 AGN, the middle panel is for the intermediate AGN type, and the lower panel is for Type-2 AGN. SEDs are colourcoded according to the contribution of the AGN to the total IR luminosity. The black solid lines at the bottom of each panel are the broad-band filters of Table 2, within which the model SEDs are integrated to generate mock photometric catalogues. The inset plots show the contribution of the three AGN components to the total SED for rrac $_{\mathrm{AGN}}=40 \%$. Red is for the dust torus emission, orange is the scattering component, and cyan the direct emission from the central AGN.

torus, emitting at shorter wavelength is completely absorbed. In the FIR rest frame, the emission of the AGN contributing to $70 \%$ of the total $L_{\mathrm{IR}}$ will dominate the emission of the host galaxy by a factor of 7. Given the ratio between the AGN emission and the host galaxy emission (Fig. 4), two bands seem to be the key to constrain the Type- 2 AGN emission, the $3-10 \mu \mathrm{m}$ rest frame and the $30-40 \mu \mathrm{m}$ rest frame as already noticed in previous works (e.g., Laurent et al. 2000). However, we note that dust emission templates are not well constrained in the $30-40 \mu \mathrm{m}$ range (Ciesla et al. 2014), so that improving them can help disentangling the AGN contribution in the FIR.

\section{Recovering the mock galaxy properties}

The SED-fitting functions of CIGALE are applied to the mock photometric galaxy catalogue of the previous section to separate the stellar emission from the AGN component and investigate how accurately stellar masses and SFRs can be determined for galaxies that host an AGN.

To perform the SED-fitting analysis, CIGALE first builds models corresponding to a range of input parameters for both the stellar and AGN components. The adopted parameters used in the fitting procedure are presented in Table 3 . The ones related to the galaxy host emission templates are selected by the experience gained from galaxy SED modelling at intermediate and high redshift using CIGALE (e.g., Giovannoli et al. 2011; Buat et al. 2012, 2014; Burgarella et al. 2013).
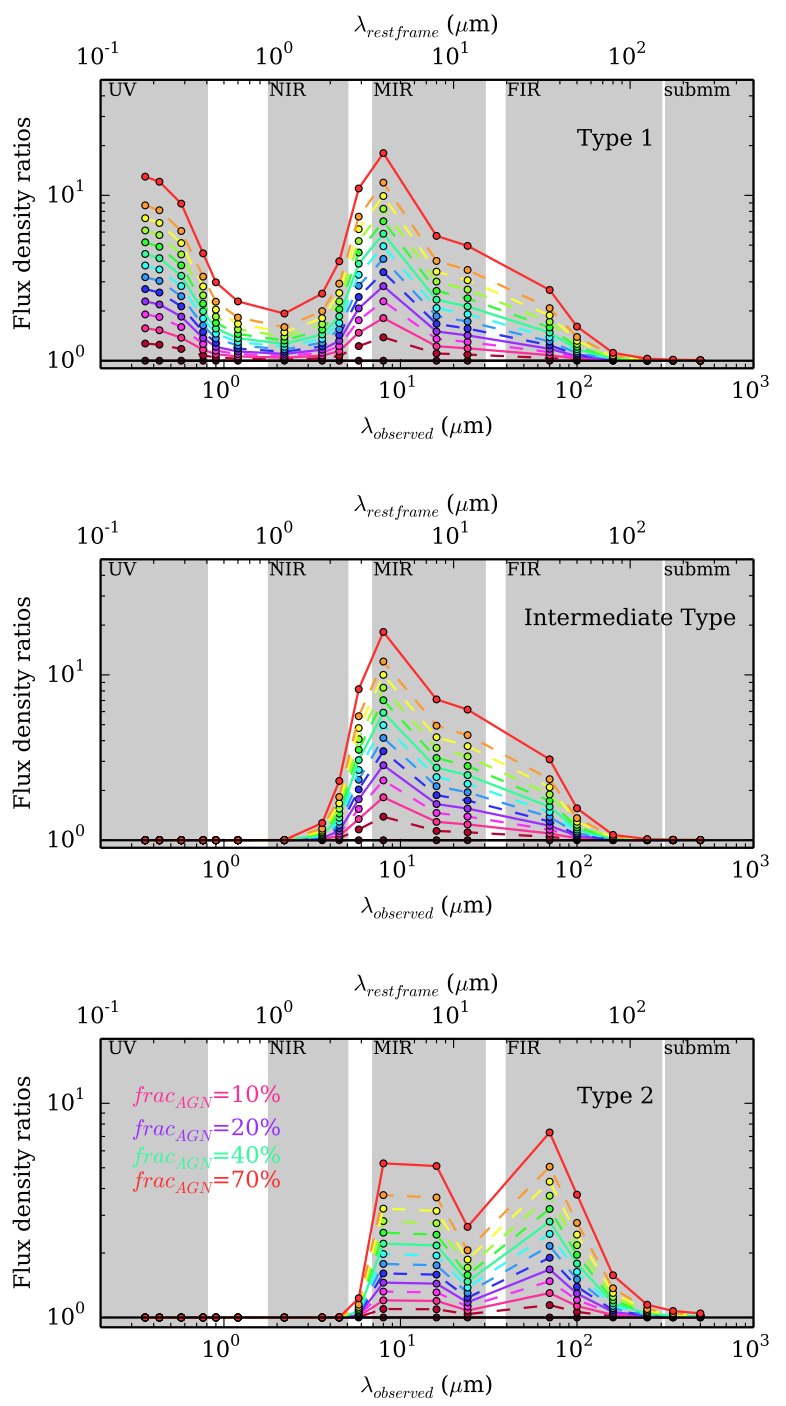

Fig. 4. The SEDs of Fig. 3 normalized to the SED with no AGN emission, i.e. rac $_{\mathrm{AGN}}=0$. The three panels correspond to one of the Fritz et al. (2006) templates presented in Table 1: upper panel is for Type-1 AGN; the middle panel corresponds to the intermediate AGN type, and the lower panel is for the Type-2 AGN. The grey regions indicate different rest frame spectral domains from UV to sub-mm. Normalized SEDs are colour-coded according to the $f_{r a c} c_{\mathrm{AGN}}$ parameter. Solid coloured lines indicate specific SEDs, i.e. where rrac $_{\mathrm{AGN}}=10 \%$ (pink), $20 \%$ (purple), $40 \%$ (green), and 70\% (red).

For the building of a galaxy template SED, it is first necessary to make some assumptions about the SFH, which will be convolved with the stellar libraries to yield galaxy SEDs. The SFH of real galaxies are expected to be highly stochastic. It is therefore impractical and probably meaningless to assume complex SFHs like those shown in Fig. 1 when fitting multi-wavelength photometric data. It is common practice instead, to assume simple functional forms, such as an exponentially decreasing SFR (1 $\tau$-dec, e.g., Ilbert et al. 2013; Muzzin et al. 2013), an exponentially increasing SFR (1-exp-ris, e.g., Pforr et al. 2012; Reddy et al. 2012), two exponential decreasing SFR laws with different e-folding times $(2 \tau$-dec, e.g., Papovich et al. 2001; Borch et al. 2006; Gawiser et al. 2007; Lee et al. 2009), a delayed SFR (e.g., Lee et al. 2010, 2011; Schaerer et al. 2013), or a lognormal SFH (Gladders et al. 2013). We consider in this work the $1 \tau$-dec, the $2 \tau$-dec, and the delayed models. First tests made with CIGALE on the lognormal SFH show that the 
Table 3. Parameter ranges used in the blind fitting procedure.

\begin{tabular}{|c|c|c|}
\hline Parameter & Symbol & Values \\
\hline \multicolumn{3}{|c|}{ Star formation history } \\
\hline Metallicity & $Z$ & 0.02 \\
\hline IMF & & Salpeter \\
\hline \multicolumn{3}{|c|}{ Double exponentially decreasing } \\
\hline$\tau$ of old stellar population models (Gyr) & $\tau_{1}$ & $1,3,5$ \\
\hline Age of old stellar population models (Gyr) & $t_{1}$ & $1,2,3,4,5$ \\
\hline$\tau$ of young stellar population models (Gyr) & $\tau_{2}$ & 10 \\
\hline Age of young stellar population models (Gyr) & $t_{2}$ & $0.01,0.03,0.1,0.3$ \\
\hline Mass fraction of young stellar population & $f_{y \mathrm{SP}}$ & $0.001,0.01,0.1,0.2$ \\
\hline \multicolumn{3}{|c|}{ Single exponentially decreasing } \\
\hline$\tau$ of stellar population models (Gyr) & $\tau$ & $0.5,1,3,5,10$ \\
\hline Age of stellar population models (Gyr) & $t$ & $1,2,3,4,5$ \\
\hline \multicolumn{3}{|c|}{ Delayed SFH } \\
\hline$\tau$ of stellar population models (Gyr) & $\tau$ & $0.5,1,3,5,10$ \\
\hline Age (Gyr) & $t$ & $4,5,5.5$ \\
\hline \multicolumn{3}{|c|}{ Dust Attenuation } \\
\hline $\begin{array}{l}\text { Colour excess of stellar continuum light for the young population } \\
E(B-V)_{*} \text { reduction factor between old and young populations }\end{array}$ & $\begin{array}{c}E(B-V)_{*} \\
f_{\text {att }}\end{array}$ & $\begin{array}{c}0.05,0.1,0.15,0.2,0.25,0.3,0.35,0.4,0.5,0.6 \\
0.44\end{array}$ \\
\hline \multicolumn{3}{|c|}{ Dust template } \\
\hline IR power-law slope & $\alpha$ & $1.5,2,2.5$ \\
\hline \multicolumn{3}{|c|}{ AGN emission } \\
\hline \multirow[t]{5}{*}{ Ratio of dust torus radii } & $R_{\max } / R_{\min }$ & 30,100 \\
\hline & $\tau_{9.7}$ & $0.3,3.0,6.0,10.0$ \\
\hline & $\beta$ & -0.5 \\
\hline & $\gamma$ & 0.00 \\
\hline & $\psi$ & $0.001,50.100,89.990$ \\
\hline Opening angle of the torus & $\theta$ & 100 \\
\hline Fraction of $L_{\mathrm{IR}}$ due to the $\mathrm{AGN}^{a}$ & $\operatorname{frac}_{\mathrm{AGN}}$ & $\begin{array}{c}-0.2,-0.15,-0.1,-0.05,0.0,0.05,0.1,0.15,0.2 \\
0.25,0.3,0.4,0.5,0.6,0.7,0.8\end{array}$ \\
\hline
\end{tabular}

Notes. ${ }^{(a)}$ We use low negative values of rrac $_{\mathrm{AGN}}$ in order to minimize a bias due to PDF analysis, as explained in Sect. 3.2.1.

associated parameters are not constrained by broad-band photometry. However, as demonstrated in Fig. 2 of Gladders et al. (2013), only specific combinations of the parameters of this SFH lead to a non-null instantaneous SFR at the age of the galaxy. Thus, we do not consider here the lognormal SFH because it provides problematic SFRs. Furthermore, since the 1-exp-ris model is recommended to only model the SFH of galaxies at $z>2$ (e.g., Maraston et al. 2010; Papovich et al. 2011; Pforr et al. 2012; Reddy et al. 2012), we do not test it in this work.

The $1 \tau$-dec is represented by the following equation:

$\operatorname{SFR}(t) \propto \exp \left(-t / \tau_{1}\right)$

where $t$ is the time and $\tau_{1}$ the e-folding time of the old stellar population. The $2 \tau$-dec is obtained by adding a late burst to the $1 \tau$-dec SFH, and thus modelled as

$\operatorname{SFR}(t)= \begin{cases}\exp -t / \tau_{1} & \text { if } t<t_{1}-t_{2} \\ \exp -t / \tau_{1}+k \times \exp -t / \tau_{2} & \text { if } t \geq t_{1}-t_{2}\end{cases}$

where $\tau_{2}$ is the e-folding time of the young stellar population, and $k$ the amplitude of the second exponential, which depends on the burst strength parameter $f_{y \mathrm{SP}}{ }^{3}$. Finally, the delayed SFH is defined as

$\operatorname{SFR}(t) \propto t \exp \left(-t / \tau_{1}\right)$

$3 f_{y \mathrm{SP}}$ is defined as the fraction of stars formed in the second burst versus the total stellar mass formed.
We assume a Salpeter IMF and the stellar population models of Maraston (2005). The metallicity is fixed to the solar one, 0.02. Dust extinction is modelled assuming the Calzetti et al. (2000) law with $E(B-V)_{*}$ in the range of values shown in Table 3 . We also assume that old stars have lower extinction than young stellar populations by a fixed factor, $f_{\text {att }}=0.44$ (Calzetti et al. 2000). The UV/optical stellar emission absorbed by dust is remitted in the IR assuming the Dale et al. (2014) templates. We emphasize that this is different from the Draine \& Li (2007) libraries used to generate the mock photometric catalogue (see Sect. 2 and Table 1). This reduces the impact on the results of using the same assumptions and templates to generate and to fit the photometry of simulated extragalactic sources.

AGN templates are also included in the fitting procedure. In Appendix A, we demonstrate that in the case of AGN hosts this is essential to minimize biases in stellar mass and SFR estimates related to contamination of the stellar light by AGN emission. Ignoring this effect results in an over-estimation of the stellar mass by up to $150 \%$ and the SFR by up to $300 \%$ depending on the spectral type and the strength of the AGN component. The parameters used to fit the AGN component are chosen based on the results of Fritz et al. (2006). However, we decide to fix the value of $\beta, \gamma$, and $\theta$, which parametrized the density distribution of the dust within the torus, with typical values found by Fritz et al. (2006) to limit the number of models. Indeed, allowing for different values of these parameters would result in degenerated model templates. 
Although the AGN parameters used to create the mock SEDs are not used in the fitting part of this work, we recognize that there could be potential problems with using the same template library to generate mock photometric data and then fitting them. As a test, we created the mock galaxies with observed AGN templates and fit them with the Fritz et al. (2006) models, and the results are discussed in Sect. 3.4. We find that our results and conclusions are robust to the set of templates used to model or fit the AGN component of the SED.

The modelled SEDs are integrated into the selected set of filters, and these modelled flux densities are then compared to the ones of the input catalogue of galaxies. The $\chi^{2}$ is computed for each galaxy in the mock photometric catalogue and for each set of model parameters. The code then builds the probability distribution function (PDF) of the derived parameters of interest (e.g. stellar mass, SFR, rrac $_{\mathrm{AGN}}$ ) based on the $\chi^{2}$ value of the fits. The output value of a parameter is the mean value of the PDF, and the associated error is the standard deviation determined from the PDF. We refer the reader to Noll et al. (2009) for more information on the Bayesian-like analysis performed by CIGALE.

To avoid any bias that could rise from our using the same tool to create and analyse the mock SEDs, we take the following precautions:

- The flux densities of the mock SEDs are perturbed by adding a noise randomly taken in a Gaussian distribution with a standard deviation of 0.1 .

- We use simple analytic SFHs in the SED-fitting procedure to reproduce GALFORM SFHs.

- Although the mock galaxies dust emission is modelled with the Draine \& Li (2007) library, we use the Dale et al. (2014) templates in the SED-fitting.

- Even though we also use the Fritz et al. (2006) library to perform the fitting, we prevent CIGALE to use the templates used in the building of the mock catalogues. We did not use a different AGN library for our fitting procedure for two reasons. The first one is that we are limited by the AGN models available in CIGALE. The second is that it has been shown in Feltre et al. (2012) that the smooth torus library of Fritz et al. (2006) is highly degenerated with the clumpy torus library of Nenkova et al. (2008). Thus using, for instance, this very different library will not affect our results.

We present in Fig. 5 examples of fits of the mock galaxies corresponding to the GALFORM SFH presented in Fig. 1 and assuming a double exponentially decreasing SFH. In Type- 1 and intermediate-type AGNs, the fits are good but we note some difficulties in reproducing measurement near the IR peak. This disagreement is attributed to compatibility problems between Dale et al. (2014) and Draine \& Li (2007) dust emission libraries in this range (Ciesla et al. 2014) that have no impact on the results of this study. For the intermediate-type AGN, Fig. 5 shows that the code uses a model with a strong silicate absorption at $9.7 \mu \mathrm{m}$. However, as shown in Fig. 3 (middle panel), the input AGN model used for the intermediate-type does not show any silicate absorption. Thus, despite the availability of models with low values of $\tau_{9.7}$ in the fitting procedure, the code does not reproduce the absence of silicate absorption of the input SED. Although these points have no impact on the result of this study, they offer a glimpse at possible problems constraining AGN templates with broad-band photometry.

The estimates of the $M_{*}$, SFR, and $f r a c_{\mathrm{AGN}}$ as a function of the contribution of the AGN are presented in Fig. 6 for the three different assumptions made on the SFH, as well as the $\chi_{\text {red }}^{2}$ distribution for each case. We discuss these results in the absence
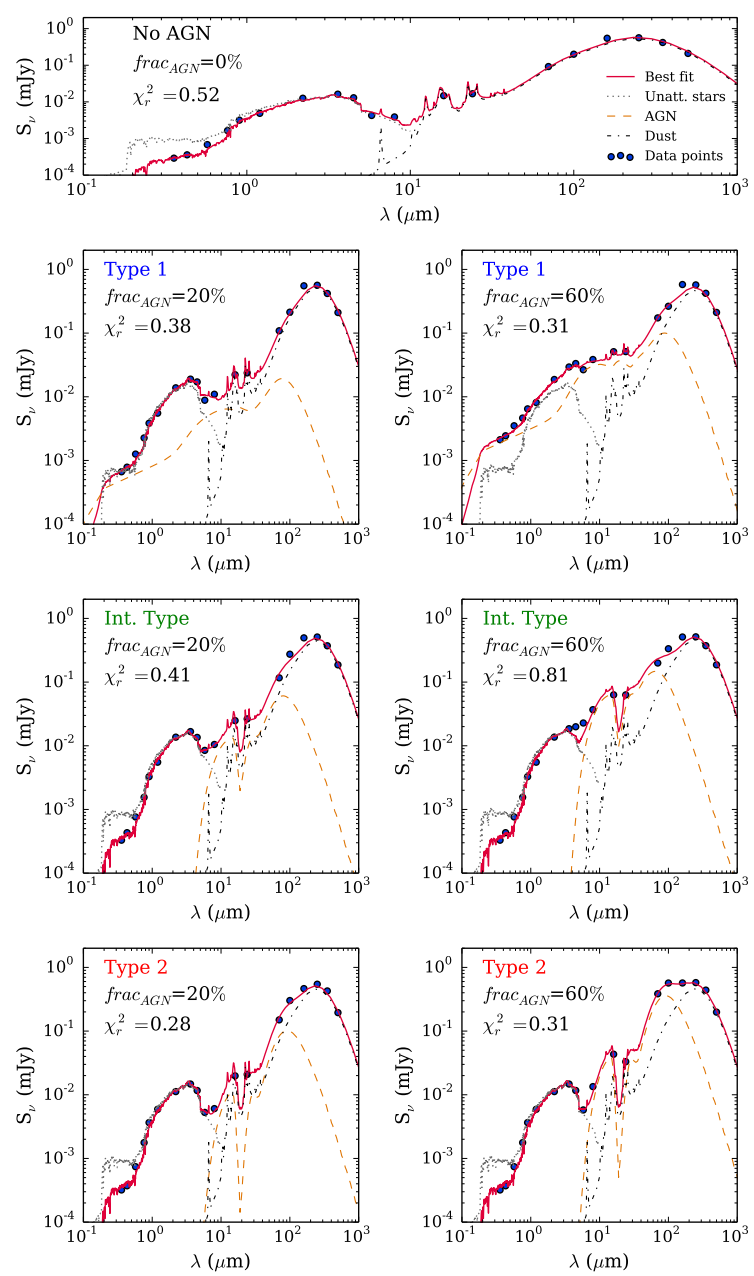

Fig. 5. Examples of fits for mock galaxies associated with one of the SFHs shown in Fig. 1. Blue points are the flux densities of the mock galaxies, and the red lines are the best fits obtained by CIGALE. In addition, we show the unattenuated stellar emission as a black dotted line, the dust emission as a black dashed-dotted line, and the total emission from the AGN as an orange dashed line. The associated reduced $\chi^{2}$ are provided. The single top panel shows the best fit in the absence of AGN emission. The two upper panels show the best fit for Type- 1 with a fraction of 20\% (left) and a fraction of $60 \%$ (right), the middle ones for the intermediate-type, and the bottom panels for the Type-2 AGN.

of AGN in Sect. 3.1, and the impact of the AGN contribution in Sect. 3.2.

\subsection{Determining stellar masses and star formation rates in simulated galaxies without AGN component}

We first explore fractional differences between the derived and input stellar masses and SFRs for mock galaxies without any AGN component included in their SEDs, i.e. the case frac $_{\mathrm{AGN}}=0$ in Fig. 6. Both parameters depend on the adopted SFH used to fit the mock galaxy multi-waveband photometry. Different functional forms for the SFH are expected to induce systematic variations in both $M_{*}$ and SFR determinations (Bell et al. 2003; Lee et al. 2009; Maraston et al. 2010; Pforr et al. 2012). Table 4 provides the fractional systematic offsets for the different SFH assumptions used in our analysis to fit the mock galaxy photometry. The model that on average provides the best agreement between the output and input $M_{*}$ and SFRs is the $2 \tau$-dec. 
L. Ciesla et al.: Derivation of stellar mass and SFR in AGN host galaxies
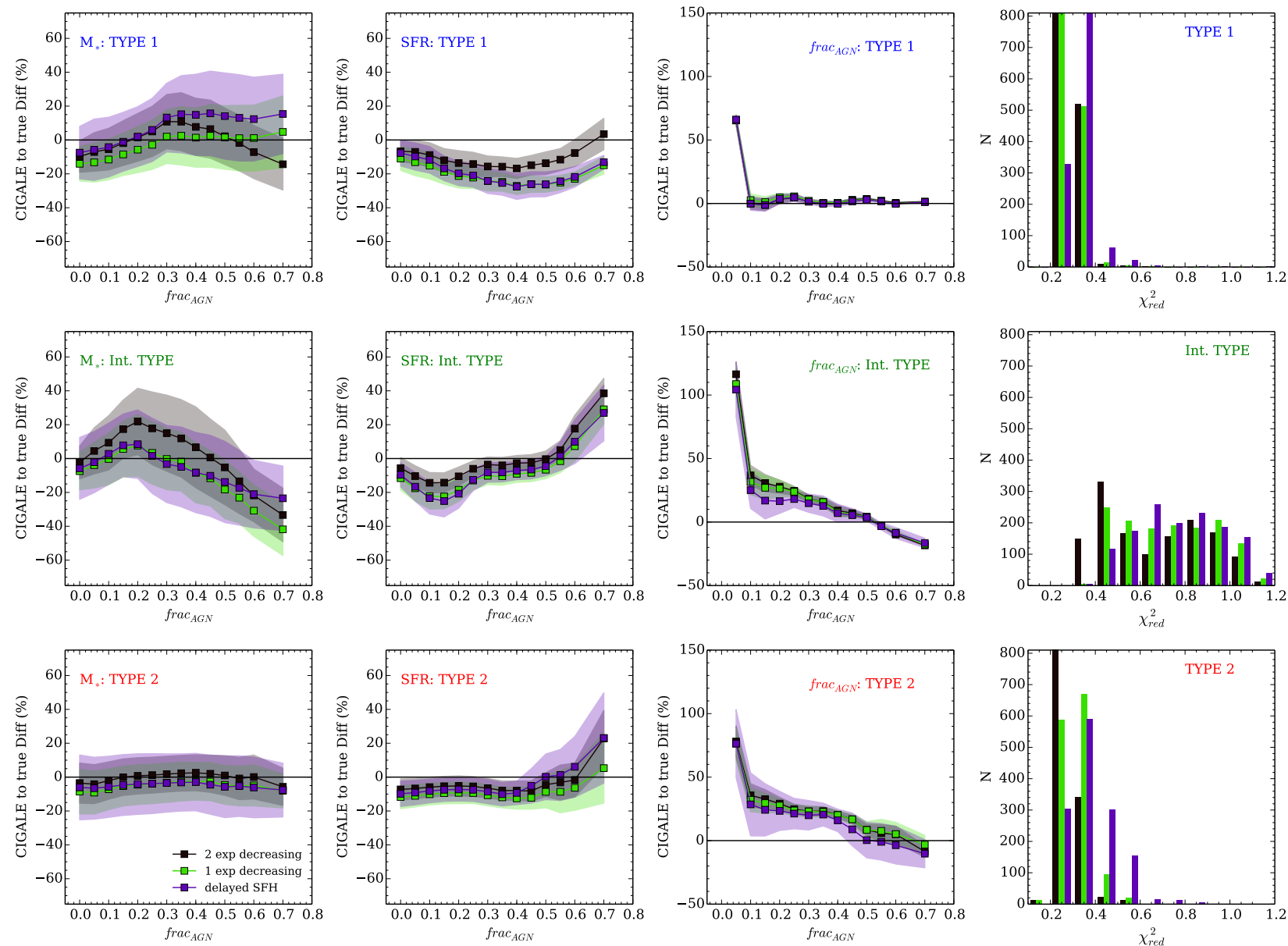

Fig. 6. Fractional difference between parameters derived from CIGALE and the simulated ones as a function of $f r a c_{\mathrm{AGN}}$, i.e. the contribution of the AGN light to the overall IR luminosity. The first column of panels plots the fractional difference in stellar mass estimates. The second

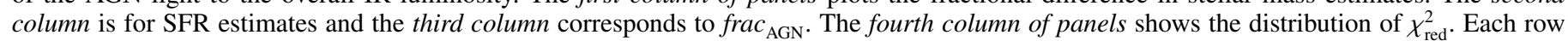
corresponds to a set of simulations that assume different input SEDs for the AGN component. From top to bottom, we present results for Type-1 AGN, intermediate-type and Type-2 AGN. Different colours in each panel correspond to different functional forms for the SFH. Black is for the $2 \tau$-dec model, green corresponds to the $1 \tau$-dec model, and purple marks the delayed SFH. Dots represent the mean value of the fractional difference at each input $f_{r a c}{ }_{\mathrm{AGN}}$, and the shaded regions show the one $\sigma$ scatter.

To compare the output parameters linked to SFH, we show in Fig. 7 five GALFORM SFHs, as well as the associated SFHs obtained from the best fit of the SED-fitting procedure. GALFORM SFHs are complex and analytical SFHs cannot reproduce the numerous star formation bursts. However, since the integral of the SFH provides the stellar mass of the galaxy, it is clear that, to recover the stellar mass, the best $1 \tau$-dec and $2 \tau$-dec models use a very small, unrealistic age. Indeed, if we fix the age of the galaxy, contrary to what is usually done in the literature when using these assumptions, by providing a narrower age range, between 4 and $5.5 \mathrm{Gyr}$ for our samples because galaxies are at $z=1$, then these two models over-estimate $M_{*}$ by 15 and $19 \%$, respectively. From the three SFHs models used in this work, the delayed SFH better reproduces the global envelope of the GALFORM SFH. As a result, this functional form yields stellar masses in reasonable agreement with the input ones and, at the same time, a galaxy formation age close to the simulated one. This is consistent with the mean SFHs of ILLUSTRIS presented in Sparre et al. (2015). Indeed, the mean and median SFHs of ILLUSTRIS sources have shapes that can typically be modelled with a delayed SFH. This result obtained from semi-analytical Models of galaxy evolution seems to agree with the conclusions of Boselli et al. (2001) based on observations of local galaxies. We thus
Table 4. Fractional differences between the derived $M_{*}$ and SFR and the input ones for the mock galaxies with $\operatorname{frac}_{\mathrm{AGN}}=0$ (no AGN contribution).

\begin{tabular}{ccccc}
\hline \hline \multirow{2}{*}{ SFH model } & \multicolumn{2}{c}{$M_{*}$} & \multicolumn{2}{c}{ SFR } \\
& Mean $(\%)$ & $\sigma(\%)$ & Mean $(\%)$ & $\sigma(\%)$ \\
\hline $1 \tau$-dec & -10.0 & 11.5 & -11.4 & 7.0 \\
$2 \tau$-dec & -5.2 & 10.2 & -6.6 & 6.2 \\
Delayed & -6.5 & 17.6 & -9.1 & 7.4 \\
\hline
\end{tabular}

conclude that a delayed SFH seems to be a more realistic assumption on the SFH of galaxies.

Several published works have studied the ability of SEDfitting techniques for retrieving the stellar mass of galaxies. They were based on mock catalogues, on SAMs, or on hydrodynamical codes, and some of them made use of the IR domain (Wuyts et al. 2009; Lee et al. 2009; Pforr et al. 2012; Pacifici et al. 2012; Mitchell et al. 2013; Buat et al. 2014). Also using GALFORM SFHs, Mitchell et al. (2013) show that a single exponentially decreasing SFH provides a good estimation of $M_{*}$ with a small offset of -0.03 dex. Using CIGALE (Buat et al. 2014) find that the output stellar mass is systematically lower by 0.07 dex 
compared to the true one. Despite the different methods used in these works, our results are in good agreement because we find an under-estimation of the stellar mass of $\sim 7 \%$ averaged over the three types of SFH.

Small differences are also found in the derivation of the SFR from the different SFH assumptions, but they generally underestimate the SFR slightly with offsets between 6.6 and $11.4 \%$ (Table 4$)$. These relatively good estimations $(<12 \%)$ obtained with the SFR are in perfect agreement with the results of Buat et al. (2014) who find that the SFR is robustly estimated, with systematic differences lower than $10 \%$, regardless the chosen SFH model as long as one IR data is available.

Another parameter that is known to be directly linked to the estimation of the stellar masses and SFR is the amount of attenuation, quantified in this work by $E(B-V)_{*}$. The link between the attenuation and the stellar mass is, however, indirect. Without a strong constraint on the dust attenuation, a degeneracy between the age of the old stellar population (which is directly linked to the stellar mass) and the attenuation appears (Pforr et al. 2012; Conroy 2013; Buat et al. 2014). We explore this bias by generating mock galaxy photometry by varying the $E(B-V)_{*}$ input value and fixing frac $_{\mathrm{AGN}}=0$. The resulting mocks catalogues are fit with a $2 \tau$-dec SFH model. The systematic offset of the derived $M_{*}$ increases by a factor of two between $E(B-V)_{*}=0.05$ and $E(B-V)_{*}=0.7$. The under-estimation on the SFR varies from $-15 \%$ to $-6 \%$ for $E(B-V)_{*}=0.05\left(A_{V}=0.2 \mathrm{mag}\right)$ to $E(B-V)_{*}=0.5\left(A_{V}=2 \mathrm{mag}\right)$. Furthermore, if, in the mock catalogues, we use a host galaxy SED with a completely obscured star formation, then the contribution of the AGN to the total IR luminosity is still recovered for high fractions, but the offset between $15 \%$ and $30 \%$ is larger by a factor of $\sim 2$. Thus the level of stellar light attenuation also plays a role in recovering the stellar mass and SFR of galaxies, as already shown in previous studies (e.g., Wuyts et al. 2009; Mitchell et al. 2013; Buat et al. 2014).

In conclusion, the $2 \tau$-dec model provides the best estimates of $M_{*}$ and SFR of the simulated galaxies, at the expense of unrealistic galaxy ages. The delayed SFH recovers the stellar mass with a mean offset of $\sim 6.5 \%$ and a mean offset on the SFR of $\sim 9 \%$, but reproduces the true SFH of the galaxies better. We use the results obtained in the absence of AGN emission as references for analysing the AGN impact on the estimation of $M_{*}$ and SFR.

\subsection{Determining stellar masses and star formation rates in AGN host galaxies}

As shown in Fig. 4, depending on its intensity, the AGN emission contaminates large parts of the SED especially the key domains used to retrieve the stellar mass and the SFR of the underlying galaxy. In this section, we discuss the ability of broad-band SEDfitting to constrain this contamination, decompose the AGN from the host galaxy light, and to determine stellar masses and SFR of AGN hosts. The results are presented in Fig. 6 for the three AGN types considered in this work, where we show the mean fractional difference for each parameter as a function of the input power of the AGN (points), as well as the $1 \sigma$ scatter.

\subsubsection{Constraining the AGN contribution}

First we explore the ability of CIGALE to constrain the fractional contribution of AGN emission to the total IR luminosity, i.e. the rac $_{\mathrm{AGN}}$ parameter. The set of panels in the third column
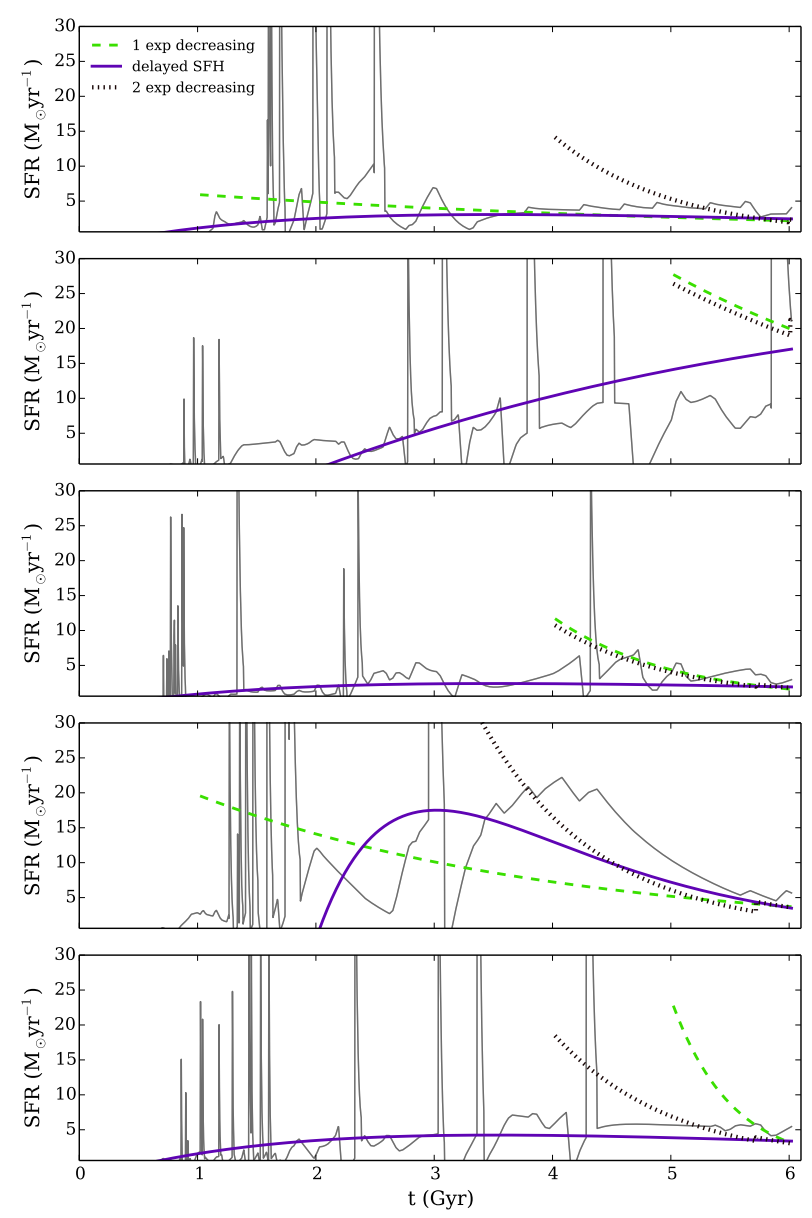

Fig. 7. Comparison between five SFHs from GALFORM and the corresponding best-fit output SFHs obtained by CIGALE. The green dashed line is for the $1 \tau$-dec model, the purple solid line corresponds to the delayed SFH, and the black dotted line to the $2 \tau$-dec model.

of Fig. 6 presents the relative difference between the output and input frac $_{\mathrm{AGN}}$ averaged over the 100 GALFORM SFHs, for the different assumptions of SFHs. The AGN contribution is almost always over-estimated for all three types, except for high fraction values $\left(\mathrm{frac}_{\mathrm{AGN}}>50 \%\right)$ for the intermediate-type and Type-2 AGNs, where it is under-estimated. Independent from the input AGN SED shape (Type-1, intermediate-type, or Type-2), below frac $_{\mathrm{AGN}}=10 \%$, there is a large over-estimation of the AGN contribution, which can reach up to $\sim 120 \%$. One explanation could be the well-known effect of the use of PDF analysis in retrieving parameters. For the lowest value of the parameter, the PDF will be truncated, and thus taking its mean value will slightly shift the output value of the parameter towards a higher value, yielding its over-estimation, as explained in Noll et al. (2009) and Buat et al. (2012). However, to prevent this effect, we provide low negative frac $_{\mathrm{AGN}}$ as well as input parameters. Without any impact on the results of the SED-fitting and the estimates of $M_{*}$ and SFR, it allows a better estimate of very low frac $_{\mathrm{AGN}}$ from the PDF analysis. Thus, the overestimation observed for very low fractions shows the difficulty that CIGALE has to distinguish between an SED without any AGN contamination and an SED with a very low contribution from the AGN ( $5 \%)$. We thus conclude that low AGN contributions are very difficult to constraint from broad-band SED-fitting with an over-estimation up to a factor of 2 for frac $_{\mathrm{AGN}}<10 \%$. At higher fractions, the over-estimation depends on the type of 

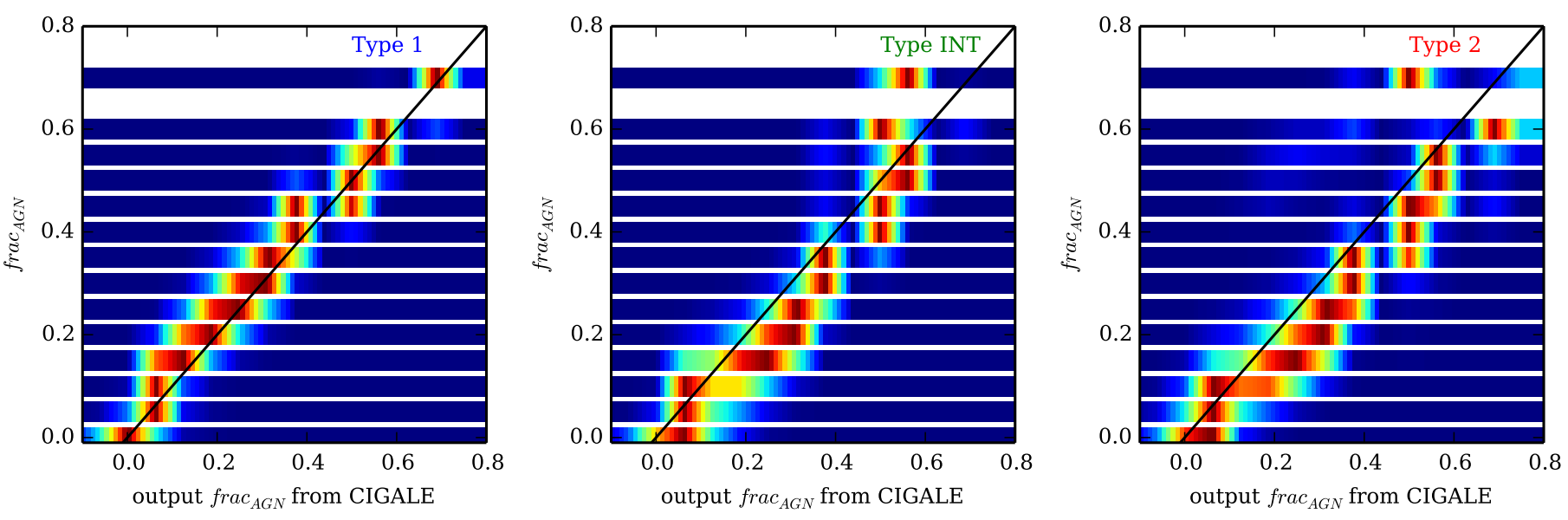

Fig. 8. Probability distribution function for the $\operatorname{frac}_{\mathrm{AGN}}$ determination of a particular GALFORM SFH. The vertical axis in all panels corresponds to different input $\mathrm{frac}_{\mathrm{AGN}}$. Each panel corresponds to a different AGN SED added to the stellar light: left is for the Type-1 AGN, the middle panel corresponds to the intermediate AGN type, and right panel plots the results for the Type-2 AGN. The colour indicates the level of probability, blue being the lowest probability and red the highest. The black solid line is the one-to-one relationship.
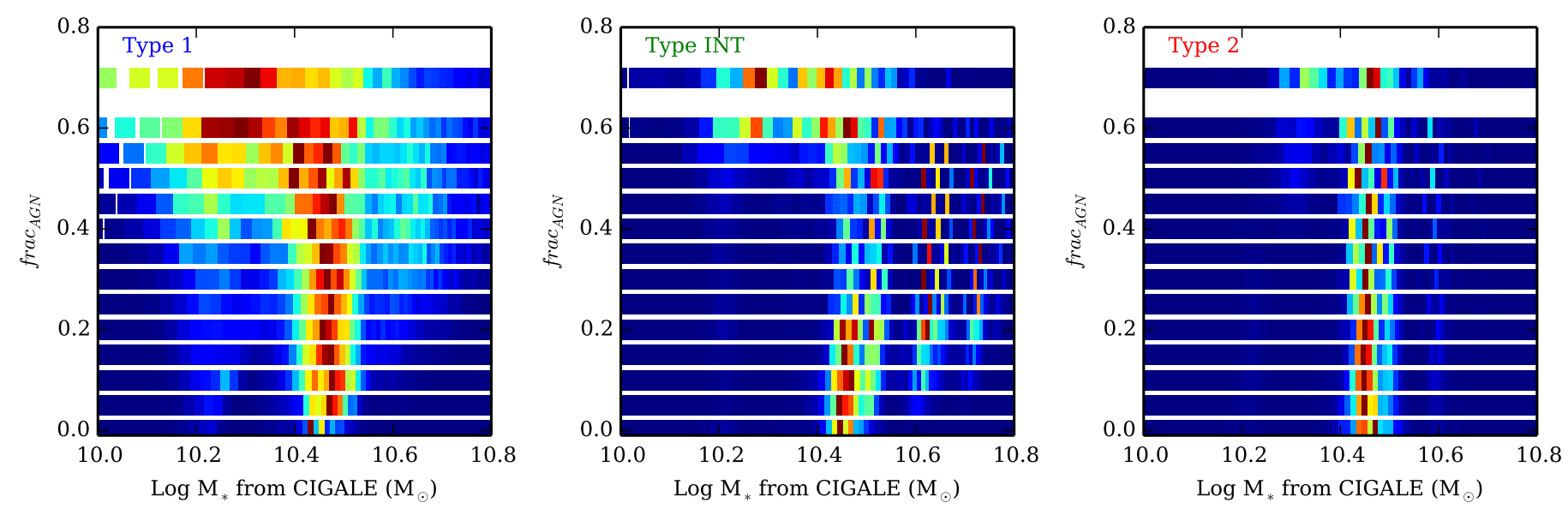

Fig. 9. Probability distribution function for the $M_{*}$ determination of a particular GALFORM SFH. The vertical axis in all panels corresponds to different input rrac $_{\mathrm{AGN}}$. Each panel corresponds to a different AGN SED added to the stellar light: left is for the Type-1 AGN, the middle panel corresponds to the intermediate AGN type, and right panel plots the results for the Type-2 AGN. The colour indicates the level of probability, blue being the lowest probability, and red the highest. The true $\log M_{*}$ for the particular simulated galaxy is 10.39 in solar units.

AGN. A Type- 1 contribution to the total $L_{\mathrm{IR}}$ is well recovered with an offset smaller than $10 \%$ for fractions higher than $10 \%$. To better understand this trend, we show in Fig. 8 (left panel) the PDF for each $f r a c_{\mathrm{AGN}}$ as seen from the top of it, for the SEDs associated to one SFH. We can see from Fig. 8 that the shape and position of the PDF do not change when rrac $_{\mathrm{AGN}}$ increases. For the intermediate-type and Type-2, an over-estimation of $30 \%$ to $40 \%$ can be expected for $f_{r a c}$ AGN between 10 and $40 \%$. Then the offset decreases at higher fractions. These effects are seen in Fig. 8, the PDF is large for low values of $\mathrm{frac}_{\mathrm{AGN}}$, and it shows a constant over-estimation up to high values of $\mathrm{frac}_{\mathrm{AGN}}$, i.e. $60 \%$.

\subsubsection{Estimating the stellar mass}

The panels in the first column of Fig. 6 plot the fractional difference between the input stellar mass of AGN hosts and the one inferred from the template fits to the mock photometry. This figure shows that the stellar masses of AGN hosts can be derived with systematic uncertainties smaller than $40 \%$ $\left(\Delta \log M_{*}<0.15 \mathrm{dex}\right)$, even in the case of Type-1 AGNs and up to rrac $_{\mathrm{AGN}}=0.7$. This underlines the importance of AGN/stellar light decomposition when fitting templates to multi-wavelength photometric data of AGN to derive properties of the underlying galaxy. If AGN templates were not included in the analysis the inferred stellar masses would be biased to high values by a factor as large as 2.5 (see Appendix A).

Careful inspection of Fig. 6 also suggests that, in the case of Type-1 AGN, the uncertainties on the estimation of $M_{*}$ as a function of Type-1 AGN fraction seem to donot depend on the assumption made on the SFH up to $\mathrm{rrac}_{\mathrm{AGN}}=0.4$. The fractional difference shows a constant increase up to rrac $_{\mathrm{AGN}}<0.4$ and then reaches a plateau for higher fractions, except for the $2 \tau$-dec model for which the offset decreases. Indeed, as we can see from Fig. 4 (upper panel), at an AGN fraction of $20 \%$, the AGN emission is higher than the host emission by a factor of 2 in the end of the NIR domain. Since NIR flux densities are known to be a proxy for the stellar mass because the emission is dominated by the old stellar population (e.g., Gavazzi et al. 1996), it is sensible to think that there is a link between the contribution of the AGN in NIR and the variations observed in Fig. 6. To understand this trend, we see in Fig. 9 (left panel) the PDF of the stellar mass for each $f r a c_{\mathrm{AGN}}$ as seen from the top of it. The PDF is very broad and is skewed toward lower values of $M_{*}$ when the frac $_{\mathrm{AGN}}$ increases. The variation in the fractional difference 

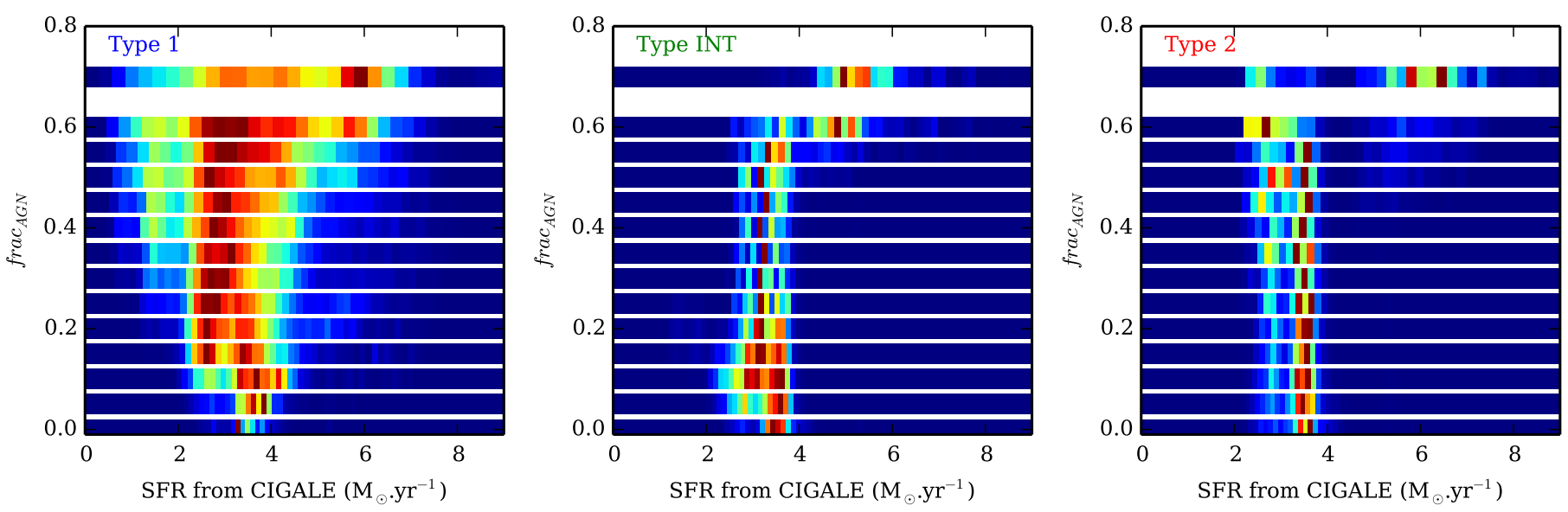

Fig. 10. Probability distribution function for the SFR determination of a particular GALFORM SFH. The vertical axis in all panels corresponds to different input $\mathrm{frac}_{\mathrm{AGN}}$. Each panel corresponds to a different AGN SED added to the stellar light: left is for the Type-1 AGN, the middle panel corresponds to the intermediate AGN type, and right panel plots the results for the Type-2 AGN. The colour indicates the level of probability, blue being the lowest probability and red the highest. The true SFR for the particular simulated galaxy is $3.3 M_{\odot} \mathrm{yr}^{-1}$.

in Type-1 AGNs depends on the contribution of the AGN to the total $L_{\mathrm{IR}}$. A very weak effect can be attributed to the assumption made on the SFH but the $1 \sigma$ scatter of each of them overlap, showing that this effect is marginal.

A systematic effect is seen in the intermediate-AGN type (Fig. 6, first column, middle panel). The offset on the stellar mass slightly increases with the contribution of AGN up to rrac $_{\mathrm{AGN}} \sim 20-30 \%$ and then decreases. This threshold of $\mathrm{frac}_{\mathrm{AGN}} \sim 20-30 \%$ corresponds to the point where the frac $_{\mathrm{AGN}}$ starts to be relatively well constrained, even if it is still over-estimated. We note that the delayed SFH appears relatively less affected showing weaker amplitude of variations with $\mathrm{frac}_{\mathrm{AGN}}$. Given the same pattern observed for three SFHs considered, the variation seen here is mostly due to the AGN emission.

For Type-2 sources, the AGN emission has no influence on the derivation of $M_{*}$ regardless of the SFH chosen. This is likely due to the high obscuration of the light emitted from the accreting supermassive black hole, which only slightly affects the observed rest-frame NIR flux of the galaxy. Indeed, it is clear from Fig. 4 that the NIR rest frame domain is not contaminated by the AGN emission. The $M_{*}$ PDF is relatively narrow and shows the offset discussed in Sect. 3.1 (Fig. 9, right panel).

\subsubsection{Estimating the star formation rate}

For all three types of AGN considered in this work, the variation in the SFR estimation as a function of AGN strength is the same for the $1 \tau$-dec, $2 \tau$-dec, and delayed SFH. This implies that these variations are entirely due to the AGN emission. For Type-1 AGNs, the under-estimation of the SFR increases with frac $_{\mathrm{AGN}}$ up to $50 \%$. Indeed, as shown in Fig. 4, the UV becomes totally dominated by the AGN emission very quickly. However, the FIR is not very affected by the AGN emission, which only dominates the $30 \mu \mathrm{m}$ rest frame emission by a factor of 3 for the highest $f_{r a c}$ AGN . This allows us to still constrain the attenuation and thus the SFR, even when there is an AGN contamination and estimate the SFR with a maximum offset of $30 \%$. The example of Fig. 10 (left panel) shows that the PDF is close to the true value, but in this case, a secondary peak arises for AGN fractions higher than $35 \%$, yielding the under-estimation.
In the case of the intermediate-AGN type and for input AGN fractions of frac $_{\mathrm{AGN}}=10 \%$ the host galaxy SFR is underestimated by a factor of $20-30 \%$. This is likely related to the over-estimation of the AGN contribution to the FIR luminosity (see Fig. 6 third column, middle panel). This results in an under-estimation of the host galaxy emission in the MIR-FIR domain, and thus an under-estimation of the SFR. However, for input rrac $_{\mathrm{AGN}}$ in the interval $25 \%$ to $50 \%$, the AGN contribution to the FIR luminosity is better constrained. As a result the inferred SFR from the SED fit is also in better agreement with the input one. For input frac $_{\mathrm{AGN}} \gtrsim 50 \%$ the opposite is happening. The AGN contribution to the FIR is under-estimated, so the host galaxy emission in the rest-frame MIR-FIR part of the SED is over-estimated. The net effect is an overall over-estimation of the SFR up to $40 \%$. This behaviour is also shown in Fig. 10 (middle panel) where the peak of the PDF is slightly shifted towards lower values up to $\mathrm{frac}_{\mathrm{AGN}}=30 \%$, then lies on the right values up to $\mathrm{frac}_{\mathrm{AGN}}=60 \%$ where the SFR starts to be over-estimated for very high fractions.

For Type-2 AGNs, we find the same pattern as in the two previous cases. The $1 \tau$-dec, $2 \tau$-dec, and delayed SFH follow the same trend. Estimation of the SFR is quite insensitive to the AGN contribution with a very weak decrease up to $\operatorname{rrac}_{\mathrm{AGN}}=$ $40 \%$. For higher fractions, the offset on the SFR measurement increases up to an over-estimation up to $20 \%$ for the $2 \tau$-dec and the delayed SFH models. A comparison between the SED with an AGN contribution of $40 \%$ and the SED of normal galaxy shows an emission that is three times higher for the AGN SED in the FIR domain, which is mandatory to have a good estimation of the SFR (Buat et al. 2014). The SFR PDF corresponding to the $2 \tau$-dec model (Fig. 10, right panel) shows two peaks, one on the right value and one slightly over-estimating the SFR. For high AGN fractions, a third peak arises that overestimates the SFR even more and skews the PDF towards higher values. However, this increase is weak for the $1 \tau$-dec SFH model yielding an over-estimation of $5 \%-10 \%$ at $\operatorname{frac}_{\mathrm{AGN}}=70 \%$.

\subsection{Impact of the photometric coverage}

The results presented in Sects. 3.1 and 3.2 were tested in the ideal case where complete photometric coverage, from UV to sub-mm rest frame, is available for each source. In this section, 

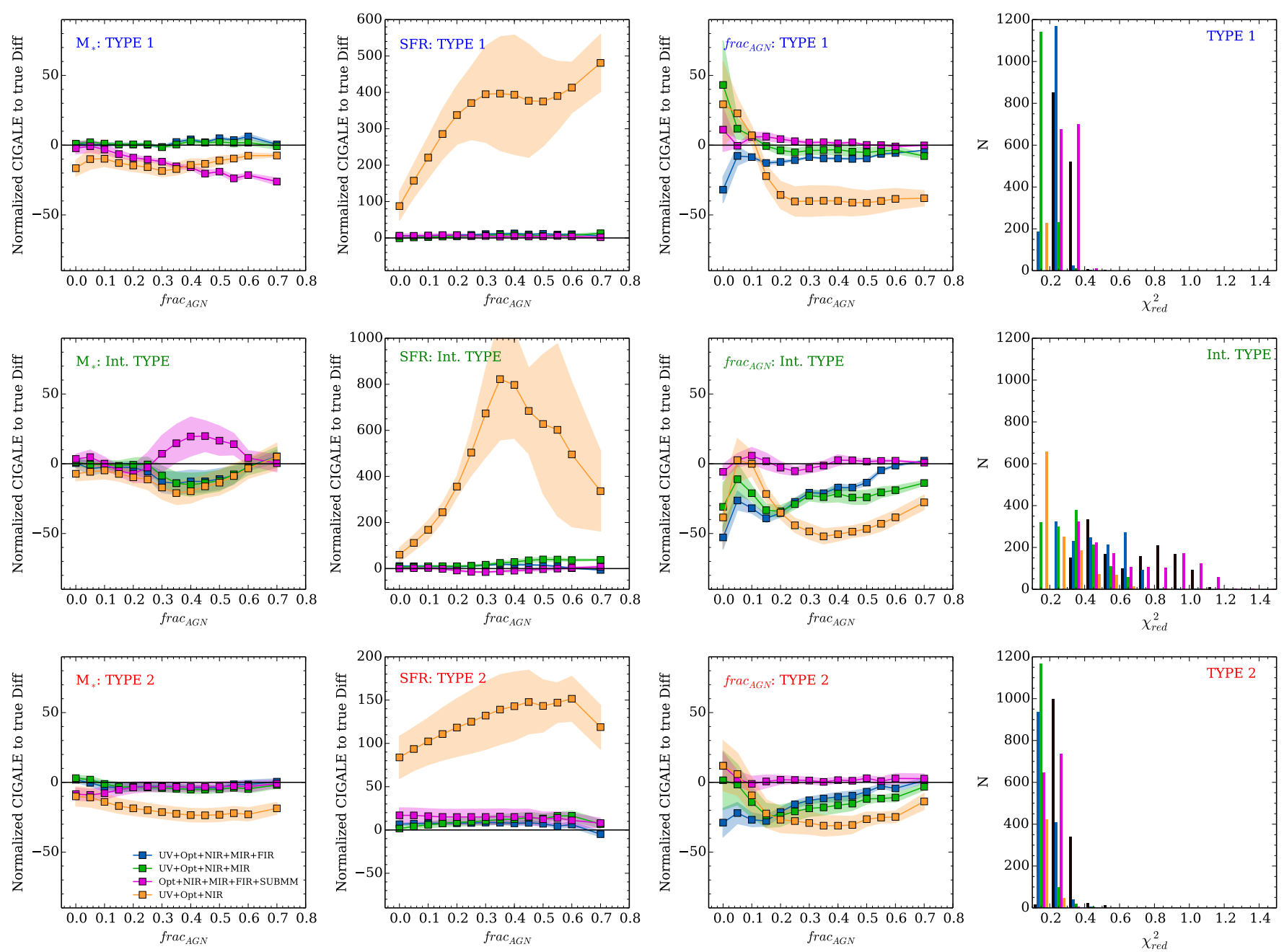

Fig. 11. Evolution of the relative difference between output parameters from CIGALE and the true ones with the input fraction of AGN for the three types of AGN considered, normalized to the values obtained with a full photometric coverage. The blue relation corresponds to a photometric coverage containing no sub-mm photometry. Green corresponds to the absence of FIR-sub-mm data. Orange corresponds to the absence of IR data. Finally, the UV was removed for the magenta relation. $\chi_{\text {red }}^{2}$ distribution are shown for every run on the fourth column, we add the distribution corresponding to the total photometric coverage in black for comparison.

we study how the lack of photometric data at different spectral bands affects the determination of the stellar mass, the SFR, and the rrac $_{\mathrm{AGN}}$ parameter. As shown in Sect. 3.1, there is no perfect SFH assumption, so we arbitrarily chose the $2 \tau$-dec SFH model. This choice does not affect the discussion because we are interested in deviations of the parameters inferred from spectral fits using incomplete broad-band coverage relative to the ideal case that all spectral bands listed in Table 2 are available. We ran our SED-fitting procedure using exactly the same parameters as in the previous sections. However, in successive runs, the input mock catalogues lacked photometry in certain broad-band filters. We explored in particular how the results on stellar mass, $\mathrm{SFR}$, and rrac $_{\mathrm{AGN}}$ change if we exclude in turn, the sub-mm rest frame (Herschel/SPIRE $350 \mu \mathrm{m}$ and $500 \mu \mathrm{m}$ ), the FIR rest frame (filters with $\lambda_{\text {mean }} \geq 24 \mu \mathrm{m}$ in Table 2 ), the mid-IR rest frame (filters with $\lambda_{\text {mean }} \geq 8 \mu \mathrm{m}$ in Table 2 ), and the UV rest frame (filters with $\lambda_{\text {mean }}<0.762 \mu \mathrm{m}$ in Table 2 ).

Figure 11 presents the results for each trial. The impact of the different spectral ranges in constraining the AGN contribution is shown in the third column of Fig. 11. The lack of UV rest-frame photometry has no impact on the determination of rrac $_{\mathrm{AGN}}$, even for Type-1 AGNs. This parameter is more sensitive to the availability of FIR and sub-mm photometry. For all input AGN spectral types and for input rrac $_{\mathrm{AGN}} \gtrsim 10-20 \%$, the lack of IR and/or sub-mm data results in a systematic under-estimation of inferred rrac $_{\mathrm{AGN}}$ up to typically $20 \%$ to $30 \%$ relative to the case of complete photometric coverage. Intermediate-type and Type-2 AGN show larger deviations compared to Type-1 AGNs. Interestingly, for input rrac $_{\mathrm{AGN}} \lesssim 10-20 \%$, the largest offset is found in the absence of sub-mm data, showing the importance for these long wavelengths in constraining the emission from dust heated by young and evolved stellar populations. The exclusion of all photometric bands above $8 \mu \mathrm{m}$ produces systematic offsets of up to $50 \%$ in the derived frac $_{\mathrm{AGN}}$. This is expected because CIGALE is based on energy balance and therefore requires long-wavelength data for optimum performance (Noll et al. 2009).

The first column of the panels in Fig. 11 shows changes in the determination of stellar mass for different sets of photometric bands. The absence of FIR and/or sub-mm has no significant impact on the derivation of the stellar mass for any of three AGN spectral types. The observed systematic variations with rrac $_{\mathrm{AGN}}$ are similar to the case of complete photometric band coverage. However, we note a small under-estimation of $\sim 10 \%$ in $M_{*}$ for intermediate type with input $f r a c_{\mathrm{AGN}}$ between 30 and $60 \%$. The lack of UV photometry has no impact 
on the determination of $M_{*}$ for Type-2 AGN. This is because in this case there is no AGN emission to contaminate the UV-toNIR bands. The absence of UV photometry however, does affect the $M_{*}$ estimates for Type-1 and intermediate-type AGN. It leads to an under-estimation of the stellar mass in Type-1 AGNs, and an over-estimation for intermediate type AGN for input rac $_{\mathrm{AGN}}=30-60 \%$. This underlines the importance of the UV for recovering the stellar mass for these types of AGNs. When no data longwards of observed $8 \mu \mathrm{m}$ are available, the stellar mass is always moderately under-estimated by up to $20 \%$ to $30 \%$, relative to the case of full photometric band coverage. This agrees with Noll et al. (2009) who find an under-estimation of 0.19 dex in the case of local normal (i.e. no AGN component) galaxies for the same combination of photometric data.

The panels in the second column of Fig. 11 shows that the photometric coverage has an impact on the determination of SFR in AGN-dominated galaxies. The strongest effects are observed in the absence of data above observed $8 \mu \mathrm{m}$. This yields large systematic errors of $150 \%$ to $800 \%$ in SFR, depending on the level of input $\mathrm{frac}_{\mathrm{AGN}}$. This agrees with Noll et al. (2009) who also find an over-estimation of the SFR by 0.83 dex for normal galaxies (i.e. no AGN component) for the same photometric data coverage. Smaller offsets in SFR estimates are found when the FIR, sub-mm and UV photometric bands are excluded. For Type- 1 and Type-2 AGNs, the absence of any of these spectral domains leads to a systematic over-estimation of $5 \%$ to $20 \%$. In the case of intermediate-type AGNs, the removal of the sub-mm leads to variations in the estimation of the SFR up to $\sim 20 \%$. The situation is worse when no FIR data is available, where the offset on the SFR increases rapidly with input rrac $_{\mathrm{AGN}}$ up to $35 \%$. The absence of UV data for intermediate-type AGNs has a peculiar effect because it starts to have an impact from frac $_{\mathrm{AGN}}=20 \%$, where the SFR is under-estimated up to $\mathrm{frac}_{\mathrm{AGN}}=60 \%$. In intermediate-types, the AGN has no impact on the UV domain, as shown in Figs. 3 and 4. Removing this range forces CIGALE to rely on the FIR to estimate the SFR, which is contaminated by the AGN emission. It is therefore difficult to constrain the SFR of objects with AGN SED components similar to the intermediate type AGN considered in this work without any UV data.

Regarding constraints on the stellar mass in the case of SEDs without AGN contribution $\left(\operatorname{rrac}_{\mathrm{AGN}}=0\right)$, very small differences, less than a few percentage points, are observed when reducing the available photometric bands. This is expected since $M_{*}$ is mainly constrained from the NIR rest frame emission. The estimation of the SFR differs by up to $\sim 10 \%$ when we remove IR and UV data. This is to be expected since these spectral domains are important for constraining the SFR.

\subsection{Impact of the AGN library}

To focus our study on the biases that AGN emission has on the estimate on $M_{*}$ and SFR, we modelled the mock galaxies using templates from the Fritz et al. (2006) library, and used the same library to perform the SED-fitting. Although we are cautious not to use the same AGN templates when simulating and fitting the mock galaxy photometry, it may be possible that the trends we observe are at least partially driven by degeneracies among the broad-band AGN SEDs of the Fritz et al. (2006) library. We explore this by using a different set of AGN templates to build the mock galaxy photometric catalogue. We selected three SEDs presented by Lusso et al. (2013), derived by Silva et al. (2004), their Seyfert 1, their mildly obscured Seyfert 2 with $\log N_{\mathrm{H}}=21.5 \mathrm{~cm}^{-2}$, and their heavily obscured Seyfert 2 with $\log N_{H}=24.5 \mathrm{~cm}^{-2}$. These templates bear similarities to the the Type-1, Intermediate type, and Type-2 Fritz et al. (2006) AGN templates defined in Table 1 . The approach described in Sect. 2.2 is followed to construct mock-galaxy and AGN composite photometry, with the only difference that the three Lusso et al. (2013) templates above are used instead of the Fritz et al. (2006) ones. The SED-fitting of the resulting mock galaxy catalogues follows the steps described in Sect. 2.2 (i.e. using Fritz et al. 2006, templates). In the same way as for Fig. 6, Fig. 12 presents the results on stellar mass, SFR, and rrac $_{\mathrm{AGN}}$ for the $2 \tau$-dec SFH model. For these parameters, the overall systematic trends in Fig. 12 are similar to those observed in Fig. 6 where the modelling of the mock galaxies is made with Fritz et al. (2006) models. We are therefore confident that our results are insensitive to the adopted AGN template library.

\section{SFR- $M_{*}$ relation}

As mentioned earlier, SED-fitting is the most popular method for deriving the physical properties of the very large galaxy samples often produced by deep wide-area extragalactic surveys. Recent studies of such large samples have shown that the majority of star-forming galaxies are known to follow an SFR- $M_{*}$ correlation, called the main sequence (MS), and galaxies that lie above this sequence are experiencing a starburst event (Elbaz et al. 2011). To understand whether if the results discussed in this work could affect the shape of the MS, we show in Fig. 13 the mock galaxies of our samples in a SFR- $M_{*}$ plot. It is known that the main sequence predicted by models currently suffers normalization problems compared to the observed relation (e.g., Mitchell et al. 2014; Furlong et al. 2014). At $z=1$ the MS predicted by GALFORM is about a factor of 2 lower than the observed MS (see Mitchell et al. 2014, for a complete discussion of this issue). The SFHs provided by GALFORM are thus probably not a perfect representation of the real $z=1$ star-forming galaxies, and it is unclear if it could affect our results. Indeed, all the models currently available suffer from the same problem. However, in this section, we focus on the effect of the AGN emission and the use of SED-fitting to retrieve the physical parameters of the galaxies on the MS.

As we discuss in Sects. 3.1 and 3.2, the choice of the SFH assumption has an impact on $M_{*}$ and SFR, and thus on the SFR$M_{*}$ correlation, as also discussed in Buat et al. (2014). The stellar masses and SFR used in Fig. 13 are those obtained from a $2 \tau$ dec SFH. The SFR- $M_{*}$ relation obtained from the Type-1 sample shows a small increase in the dispersion that is slightly shifted towards higher stellar masses, owing to the offset on the stellar mass obtained for high fractions of AGN discussed in Sect. 3.1 (Fig. 13, upper left panel). Thus, the increasing offset on the stellar mass estimation for Type-1 AGNs has a small impact on the SFR- $M_{*}$ relation. For the intermediate-type, a shift due to the stellar mass offset is observed, but in addition there is an increase in the MS dispersion owing to the variation in the offsets with the AGN contribution. Low fractions shift the relation towards higher $M_{*}$ and the higher fractions towards lower masses, thus increasing the dispersion of the relation. However, the Type-2 SFR $-M_{*}$ relation does not show a significant change because the $M_{*}$ estimation is totally independent of rac $_{\mathrm{AGN}}$. Furthermore, the under-estimation observed for low AGN fraction in the estimation of the SFR does not seem to affect the MS of the Type-2 sample.

The lower panels of Fig. 13 present the same results but for the specific SFR (sSFR). In contrast to the Type-1 and Type-2 samples, the intermediate-type sample displays a correlation between the fractional difference between the mock and output 

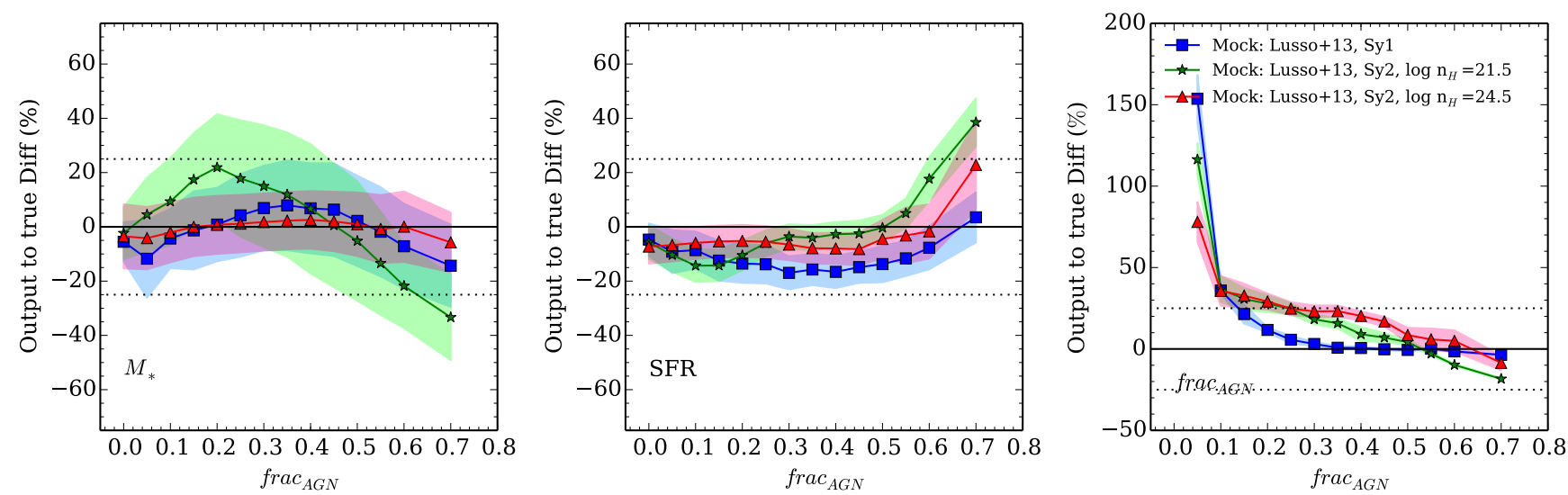

Fig. 12. Comparison between the output values of $M_{*}$, SFR, and $f r a c_{\mathrm{AGN}}$ and the true ones in the case where the AGN emission of the mock galaxies is modelled using Lusso et al. (2013) templates. The SED-fitting is made using the $2 \tau$-dec SFH models and the Fritz et al. (2006) models. The results for the Type-1 sample are in blue, for the intermediate-type sample are in green, and for the Type- 2 sample in red.
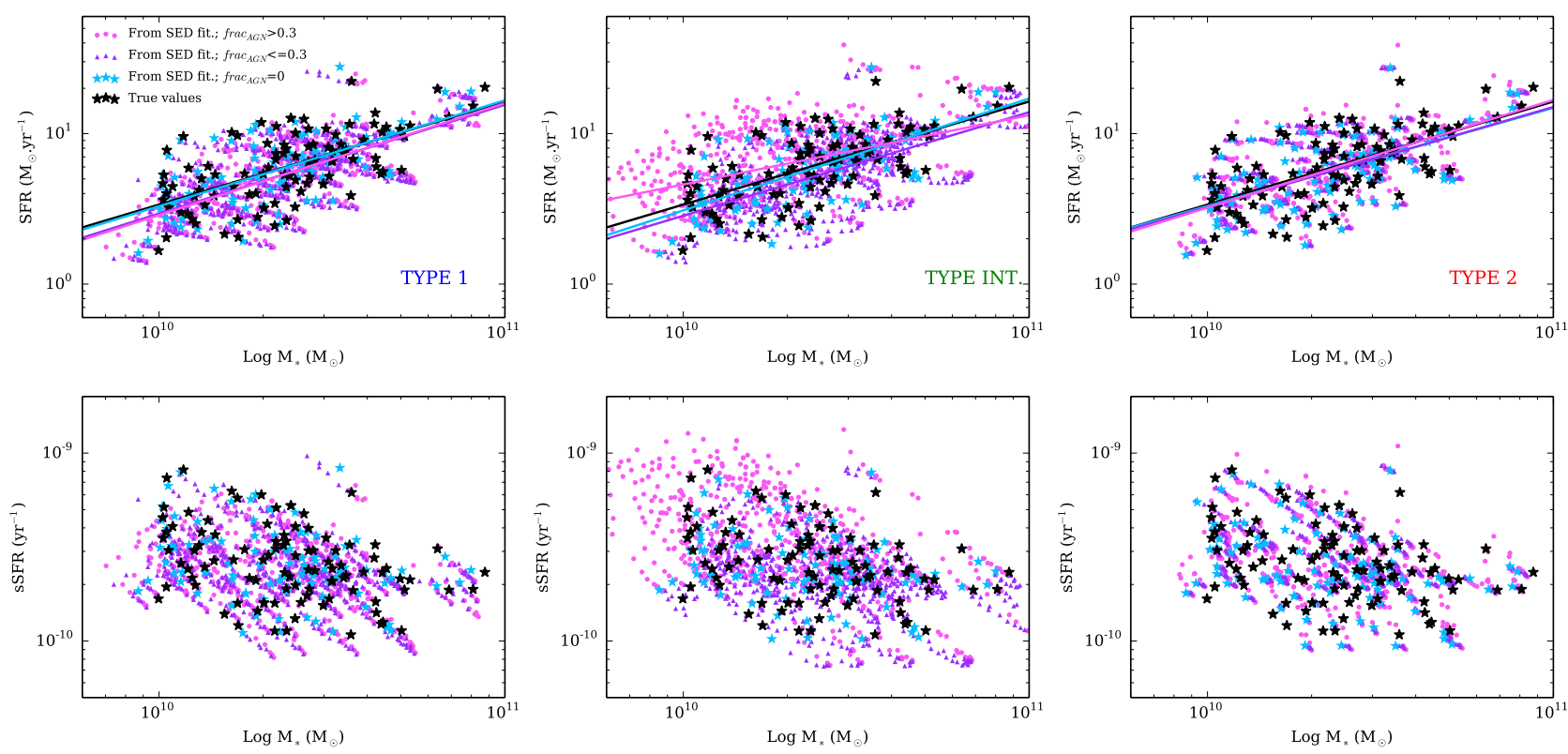

Fig. 13. Top panels: $S F R-M_{*}$ diagram for the Type-1 sample (left), the intermediate-type (middle), and the Type-2 sample (right) obtained when using the $2 \tau$-dec SFH model for the fitting. Black stars are the true values of the SFR and $M_{*}$ of the mock galaxies. Cyan stars are the galaxies without any AGN component ( $\mathrm{rac}_{\mathrm{AGN}}=0$ ), purple triangle are galaxies with an AGN contribution lower than $30 \%$, and pink circle galaxies with an AGN contribution higher than $30 \%$. Colored-lines are the linear fit corresponding to each subsample. Bottom panels: sSFR - M* diagram for the Type-1 sample (left), the intermediate-type (middle), and the Type-2 sample (right).

parameter values and $\mathrm{frac}_{\mathrm{AGN}}$. The sample without $\mathrm{AGN}$ is biased towards low sSFR and higher stellar mass and increase towards higher sSFR and lower stellar mass with $f_{r a c} c_{\mathrm{AGN}}$. The Type-1 sample only shows an under-estimation of the SSFR, as well as the Type-2 sample where the under-estimation is, however, weaker.

As these results depend on the assumption made on the SFH, we provide in Table 5 the linear coefficients of the best fits of the SFR- $M_{*}$ such as

$$
\log S F R=a \times \log M_{*}+b .
$$

We provide these coefficients for the "true" sample, for the sample containing no AGN, and for the Type-1, int.-type, and Type-2 AGN samples, in all three SFH cases. In no case were we able to recover the true slope of the MS, but the closest value is obtained for the $2 \tau$-dec SFH ( 0.70 when the true slope is 0.68$)$ which is the SFH assumptions providing the best estimation of both $M_{*}$
Table 5. Variations in the $S F R-M_{*}$ correlation depending on the SFH.

\begin{tabular}{cccccc}
\hline \hline Coefficients & True & No AGN & Type-1 & Int.-type & Type-2 \\
\hline \multicolumn{7}{c}{$1 \tau$-dec } \\
$a$ & 0.68 & 0.62 & 0.72 & 0.47 & 0.63 \\
$b$ & -6.32 & -5.66 & -6.84 & -4.10 & -5.85 \\
\hline \multicolumn{6}{c}{$2 \tau$-dec } \\
$a$ & 0.68 & 0.70 & 0.74 & 0.66 & 0.81 \\
$b$ & -6.32 & -6.51 & -6.91 & -6.11 & -7.73 \\
\hline \multicolumn{7}{c}{ Delayed } \\
$a$ & 0.68 & 0.77 & 0.56 & 0.59 & 0.74 \\
$b$ & -6.32 & -7.16 & -5.16 & -5.37 & -6.89 \\
\hline
\end{tabular}

and SFR. The larger the offset on the stellar mass estimation, the larger the difference to the true MS slope. It seems that the 
SFR- $M_{*}$ relation is more sensitive to the estimation of the stellar mass than of the SFR. The type of AGN has an influence on the MS slope for all three SFHs with a difference up to $\sim 32 \%$.

\section{Comparison against IR-only SED decomposition}

In this section, we use our mock catalogues to test another method of disentangling the AGN emission from the host galaxy in the IR, DECOMPIR ${ }^{4}$ (Mullaney et al. 2011). The choice of DECOMPIR is driven by its being a popular code for deriving the IR properties of AGN host galaxies and is publicly available. The aim here is to not compare the results obtained from CIGALE and DECOMPIR because they are two very different methods. Indeed, results from CIGALE are based on energy-balance SED-fitting using multi-wavelength data from UV to sub-mm, whereas DECOMPIR is based only on the IR. DECOMPIR uses a set of observed templates for the host and the AGN. The hostgalaxy templates are derived by using the Brandl et al. (2006) SB sample, as well as four galaxies taken in the Revised Bright Galaxy Sample. These host templates are grouped into five different averages, spanning a wide range of possible observed SB IR SEDs. The AGN template is defined by effectively subtracting these host templates from the IR SEDs of local AGNs (Mullaney et al. 2011).

To determine how successfully DECOMPIR separates the AGN component from the host emission, and thus estimates the SFR, we perform SED-fitting with the five different SB templates and an AGN component using MIPS $24 \mu \mathrm{m}$ to SPIRE $500 \mu \mathrm{m}$ data, corresponding to 12 to $250 \mu \mathrm{m}$ rest frame. For each mock galaxy, we also fit the IR SEDs, using only the five different SB components, i.e. without allowing any AGN contribution. This last test is performed to examine the value of adding an AGN component to the fit. As a result, each mock galaxy is tested with ten different configurations (i.e. 5 double components and 5 single components) for which $\chi^{2}$ are derived.

We then use an Akaike information criterion (AIC) to select the best model, regardless the number of components used to fit the data. The AIC is comparable to the more popular Bayesian information criterion (BIC). Although both criteria are derived in the same way, the main difference comes from the prior used that is inversely proportional to the number of models for the $\mathrm{BIC}$, and a decreasing function of the number of models for the AIC. However, some advanced studies that compare both criteria, showed that the AIC is more accurate in model selection than the BIC (see Burnham \& Anderson 2004; and Yang 2005, for detailed comparisons). For each mock galaxy, we submit the ten different $\chi^{2}$ to the AIC. We find that for the Type-1, intermediate-type, and Type- 2 AGNs, the AGN components improves the fit for 36,21 and $47 \%$ of the galaxies, respectively. For all of the other galaxies, regardless of the type, a SB template is enough. We then estimate the AGN fraction, the $L_{\mathrm{IR}}$, and the uncontaminated SFR using Kennicutt (1998).

Figure 14 shows the results, i.e. the variation in the estimation of the AGN contribution, the SFR, and the $L_{\mathrm{IR}}$ with the AGN contribution using DECOMPIR. We present the estimate of the $L_{\mathrm{IR}}$ because the SFR is determined from the simple conversion of $L_{\mathrm{IR}}$ using Kennicutt (1998). The AGN contribution is always under-estimated (except for one point corresponding to $\mathrm{frac}_{\mathrm{AGN}} \sim 20 \%$ in the Type-2 sample). Confirming what observed with the CIGALE results, low fractions of AGN are

\footnotetext{
4 The code is publicly available at https://sites.google.com/ site/decompir/
}

difficult to constrain. The Type-1 AGN contribution is underestimated by at least $25 \%$, whereas intermediate-type rrac $_{\mathrm{AGN}}$ is closer to the true value with an offset comprised between 15 and $50 \%$. The variation of the estimation of the AGN contribution of the Type- 2 sample is, however, peculiar since it is not monotonic. Like the results obtained with CIGALE, the SFR is slightly under-estimated but recovered well whatever the fraction of AGN for the Type- 1 and intermediate-type sample, and up to $70 \%$ for the Type- 2 sample. This is linked to the good recovery of the $L_{\mathrm{IR}}$ with a small over-estimation of a few percentage points in the absence of AGN, which does not evolve with rac $_{\mathrm{AGN}}$ for the intermediate-type sample, slightly increases up to $\sim 15 \%$ for the Type- 1 sample, and follows the peculiar behaviour observed for the estimation of $f \mathrm{Fc}_{\mathrm{AGN}}$ for the Type-2 sample. However, despite problems in recovering the right contribution of the AGN, the SFR of Type-1 and intermediate-type AGNs are fully recovered.

The estimate of the SFR and $L_{\mathrm{IR}}$ of our Type-2 AGN sample by DECOMPIR is problematic for fractions greater than $40 \%$. One explanation is that Type-2 templates from Fritz et al. (2006) predict IR SED that are cooler than what was empirically obtained by Mullaney et al. (2011). Thus, when DECOMPIR tries to fit the Type- 2 catalogue created from Fritz et al. (2006) models, the models attribute the excess of FIR emission produced by the Type-2 AGN from Fritz et al. (2006) to star formation, leading to the offset on the $L_{\mathrm{IR}}$ that we see in Fig. 14. To test this point, we ran DECOMPIR on the mock catalogue obtained with the Type-2 AGN template of Lusso et al. (2013) used in Sect. 3.4. The results are presented in Fig. 14. The SFR and $L_{\mathrm{IR}}$ are no longer over-estimated. DECOMPIR provides a good estimate of the SFR with a small under-estimation between 10 and $20 \%$, as well as for the $L_{\mathrm{IR}}$ with an under-estimation up to $20 \%$ for the highest fraction. Interestingly, the estimate on the contribution of the AGN to the $L_{\mathrm{IR}}$ is well constrained and is similar to what was obtained with CIGALE, i.e an over-estimation of low fractions of about $50 \%$.

\section{Conclusions}

We have simulated realistic SEDs of Type-1, intermediate-type, and Type-2 AGNs hosts in order to evaluate the impact of the AGN emission on the host SED, and estimate the ability of SED-fitting code to retrieve the physical properties of the galaxy. CIGALE was used to model the SEDs from SFHs provided by the SAM GALFORM code. Three samples were built, one with the emission of a Type-1AGN, one with the emission of an intermediate-type AGN, and one with the emission of a Type-2 AGN, varying their contribution through the rac $_{\mathrm{AGN}}$ parameter. We used the SED-fitting function of the recently updated CIGALE model to derive the stellar mass, star formation rate, and contribution of the AGN of each mock galaxy, assuming three popular shapes of SFH: $1 \tau$-dec, $2 \tau$-dec, and a delayed SFH.

In the absence of an AGN contribution, i.e. in normal galaxies, the SED-fitting of our mock samples shows that all the three SFHs considered in this work provide good estimations of the stellar mass within $\sim 10 \%$. The best estimates are obtained using the $2 \tau$-dec model but at the expense of realistic ages of the stellar population, whereas the delayed model provide both. Star formation rates are well recovered within $12 \%$.

For AGNs, the SED-fitting of our mock samples shows that

- Stellar masses are overall well recovered with systematics up to $40 \%(0.17 \mathrm{dex})$ or better, depending on the frac $_{\mathrm{AGN}}$ and spectral shape of the AGN component. This result is rather 

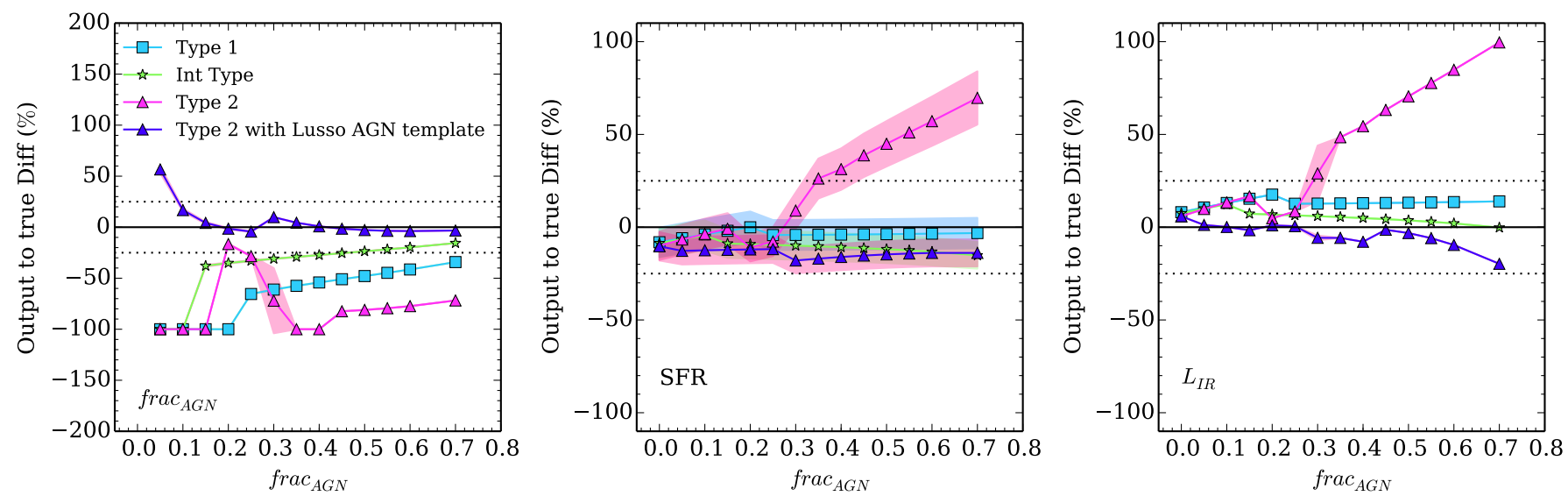

Fig. 14. Relative difference between the $f_{r a c_{\mathrm{AGN}}}$ (left panel) and the SFR (right panel) obtained by DECoMPIR and the true values. Cyan squares, green triangles, and magenta triangles are Type-1, intermediate-type, and Type-2 outputs from DECOMPIR, respectively. The purple triangles present the results provided by DECOMPIR for the Type-2 mock catalogue obtained using the Lusso et al. (2013) templates. The dotted lines indicate the $25 \%$ difference.

insensitive to the photometric bands available as long as UVMIR data are available. So $M_{*}$ is the most robust parameter that one can constrain for AGN host galaxies via broad-band photometric decomposition.

- The SFR suffers from systematic uncertainties up to $40 \%$ to $50 \%$ or better as long as FIR/sub-mm data are available. Data sets that are limited to the MIR cannot be used to constrain the SFR of AGN hosts.

- AGN/galaxy decomposition based on broad-band photometry can lead to significant over-estimation of the AGN fraction, hence the inferred AGN luminosity in the case of weak AGN.

We note the need for UV rest frame data to constrain the stellar mass and SFR of Type- 1 and intermediate-type AGNs.

The AGN emission has an influence on the slope of the MS depending on the SFH assumptions used for the SED-fitting and the type of AGN. Since the AGN contribution can slightly bias the estimates of $M_{*}$ and SFR, the variety of AGN types and AGN intensities increases the dispersion of the MS. Furthermore, the SFR- $M_{*}$ relation is more sensitive to the estimation of the stellar mass than of the SFR.

Finally, we used our mock samples to test a popular method used to disentangle the AGN emission from the host emission in the IR, DECOMPIR (Mullaney et al. 2011), and find that, when using the mock catalogues built from Fritz et al. (2006) AGN templates, frac $_{\mathrm{AGN}}$ is always under-estimated but the SFR is recovered well for Type-1 and intermediate-types of AGN, and over-estimated in Type-2 AGNs when frac $_{\mathrm{AGN}}>35 \%$. The over-estimation of the SFR of Type-2 AGNs is due to the FIR prediction of Type-2 AGN from Fritz et al. (2006) which is colder than what was observed by Mullaney et al. (2011). When using the Type-2 mock catalogue built from Lusso et al. (2013), DECOMPIR recovers the SFR and $L_{\mathrm{IR}}$ wellwith an underestimation up to $20 \%$.

In this work, we have validated the use of broad-band SED-fitting methods to derive the stellar mass and SFR of AGN host galaxies, as well as the contribution of this AGN to the host-galaxy SED. This analysis provides the foundation for future AGN multi-wavelength studies using CIGALE. Indeed, in the near future, the eROSITA X-ray telescope will provide observations of about three million X-ray detected AGNs, as well as samples of tens of thousands of obscured AGNs. These data will provide accurate studies of the relationship between the accretion on the SMBH and the host-galaxy properties. Multiwavelength analysis using SED-fitting will thus be needed to derive these properties.

Acknowledgements. We thank the referee for the detailed comments that helped to improve the paper. L.C. warmly thanks M. Boquien, Y. Roehlly and D. Burgarella for developing the new version of CIGALE on which the present work relies. L.C. also thanks James Mullaney for useful discussions and suggestions, and Elisabeta Lusso for providing her SED templates. This work benefited from the THALES project 383549 that is jointly funded by the European Union and the Greek Government in the framework of the programme "Education and lifelong learning".

\section{Appendix A: The need for an AGN component to perform the SED-fitting}

In this work, we use a SED-fitting procedure allowing the possibility of using an AGN component. To understand how omitting to use this component would affect the estimate of the stellar mass and SFR of the host galaxies, we perform a SED-fitting, using the $2 \tau$-dec SFH model, and not allowing for the use of AGN templates.

Figure A.1 presents the fractional difference between the output parameters and the true ones as a function of the input frac $_{\mathrm{AGN}}$. The estimate of the stellar mass of the Type-2 sample is not perturbed by the AGN, as we discuss in this work. The NIR bands are not affected by the AGN emission, thus the stellar mass is well recovered, with or without the use of an AGN component. However, Type- 1 and intermediate-type SEDs are rapidly affected by the AGN emission, and $M_{*}$ is over-estimated by $50 \%$ at rrac $_{\mathrm{AGN}}=10 \%$. This over-estimation reaches $150 \%$ for $\mathrm{frac}_{\mathrm{AGN}}=70 \%$ in the case of Type-1 AGNs.

The SFR in intermediate-type and Type-2 AGNs is overestimated by $20 \%$ up to frac $_{\mathrm{AGN}}=30-40 \%$. This overestimation increases for higher fractions, especially in Type-2 AGNs where it can reach $100 \%$ The case of Type-1 AGNs is more critical, the SFR is rapidly and strongly over-estimated up to $300 \%$ at rac $_{\mathrm{AGN}}=70 \%$.

The $\chi_{\text {red }}^{2}$ distributions shows a higher value of the $\chi_{\text {red }}^{2}$ by a factor of $\sim 10$ compared to the distribution from the fitting using the AGN component (Fig. 6).

These results show that not taking an AGN component into account when performing broad-band SED-fitting of AGN host 

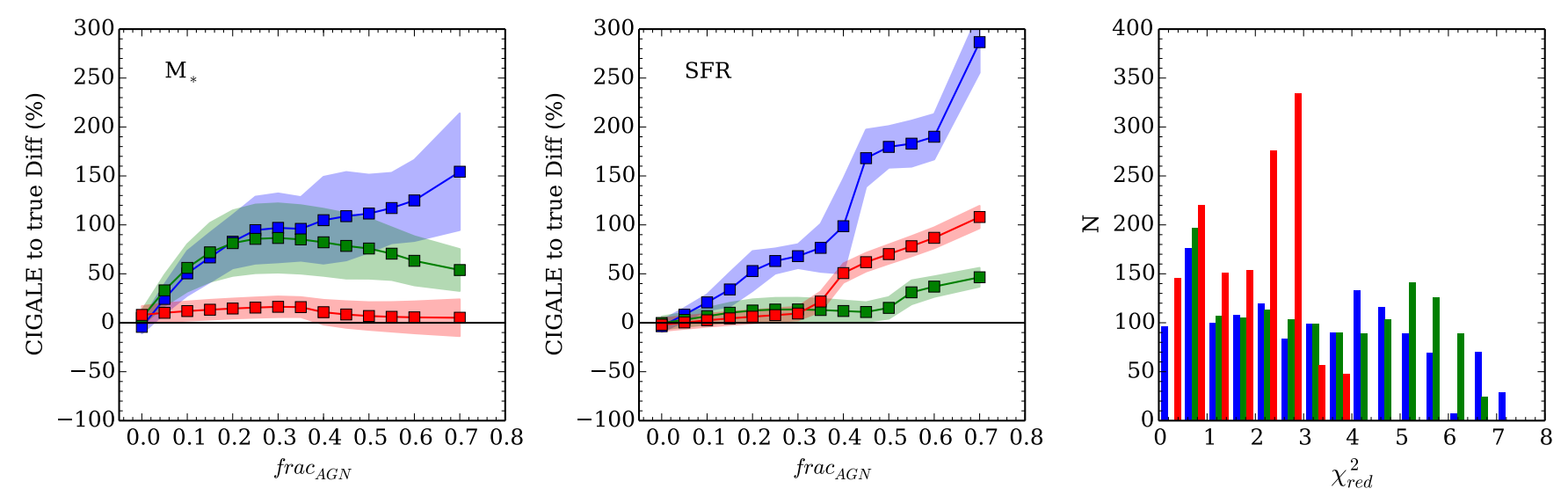

Fig. A.1. Evolution of the CIGALE to true relative difference of $M_{*}$ and SFR with the fraction of AGN in the case where the AGN component is not taken into account in the fitting procedure. Left panel shows the results for the stellar mass, middle panel for the SFR, and the right panel the $\chi_{\text {red }}^{2}$ distribution. Blue, green, and red data are for Type-1, intermediate-type, and Type-2 AGNs, respectively.

galaxies results in strong biases in determining physical properties such as the stellar mass and the star formation rate.

\section{References}

Aird, J., Coil, A. L., Moustakas, J., et al. 2012, ApJ, 746, 90 Azadi, M., Aird, J., Coil, A., et al. 2014 [arXiv: 1407.1975] Baldry, I. K., Glazebrook, K., Brinkmann, J., et al. 2004, ApJ, 600, 681 Baldry, I. K., Balogh, M. L., Bower, R. G., et al. 2006, MNRAS, 373, 469 Banerji, M., Glazebrook, K., Blake, C., et al. 2013, MNRAS, 431, 2209 Baugh, C. M., Lacey, C. G., Frenk, C. S., et al. 2005, MNRAS, 356, 1191 Bell, E. F., McIntosh, D. H., Katz, N., \& Weinberg, M. D. 2003, ApJS, 149, 289

Bell, E. F., Wolf, C., Meisenheimer, K., et al. 2004, ApJ, 608, 752

Bell, E. F., van der Wel, A., Papovich, C., et al. 2012, ApJ, 753, 167

Benson, A. J., \& Bower, R. 2010, MNRAS, 405, 1573

Blustin, A. J., Page, M. J., Fuerst, S. V., Branduardi-Raymont, G., \& Ashton,

C. E. 2005, A\&A, 431, 111

Bongiorno, A., Merloni, A., Brusa, M., et al. 2012, MNRAS, 427, 3103 Boquien, M., Buat, V., \& Perret, V. 2014, A\&A, 571, A72

Borch, A., Meisenheimer, K., Bell, E. F., et al. 2006, A\&A, 453, 869

Boselli, A., Gavazzi, G., Donas, J., \& Scodeggio, M. 2001, AJ, 121, 753

Bower, R. G., Benson, A. J., Malbon, R., et al. 2006, MNRAS, 370, 645

Brandl, B. R., Bernard-Salas, J., Spoon, H. W. W., et al. 2006, ApJ, 653, 1129

Bruzual, G., \& Charlot, S. 2003, MNRAS, 344, 1000

Buat, V., Noll, S., Burgarella, D., et al. 2012, A\&A, 545, A141

Buat, V., Heinis, S., Boquien, M., et al. 2014, A\&A, 561, A39

Burgarella, D., Buat, V., Gruppioni, C., et al. 2013, A\&A, 554, A70

Burnham, K. P., \& Anderson, D. R. 2004, Sociological Methods and Research, 33,261

Calzetti, D., Armus, L., Bohlin, R. C., et al. 2000, ApJ, 533, 682

Casey, C. M. 2012, MNRAS, 425, 3094

Cicone, C., Feruglio, C., Maiolino, R., et al. 2012, A\&A, 543, A99

Cicone, C., Maiolino, R., Sturm, E., et al. 2014, A\&A, 562, A21

Ciesla, L., Boquien, M., Boselli, A., et al. 2014, A\&A, 565, A128

Cole, S., Lacey, C. G., Baugh, C. M., \& Frenk, C. S. 2000, MNRAS, 319, 168

Conroy, C. 2013, ARA\&A, 51, 393

Conroy, C., Gunn, J. E., \& White, M. 2009, ApJ, 699, 486

Crenshaw, D. M., Kraemer, S. B., \& George, I. M. 2003, ARA\&A, 41, 117

Dale, D. A., \& Helou, G. 2002, ApJ, 576, 159

Dale, D. A., Helou, G., Magdis, G. E., et al. 2014, ApJ, 784, 83

Di Matteo, T., Springel, V., \& Hernquist, L. 2005, Nature, 433, 604

Draine, B. T., \& Li, A. 2007, ApJ, 657, 810

Elbaz, D., Daddi, E., Le Borgne, D., et al. 2007, A\&A, 468, 33

Elbaz, D., Dickinson, M., Hwang, H. S., et al. 2011, A\&A, 533, A119

Faber, S. M., Willmer, C. N. A., Wolf, C., et al. 2007, ApJ, 665, 265

Fabian, A. C. 1999, MNRAS, 308, L39

Feltre, A., Hatziminaoglou, E., Fritz, J., \& Franceschini, A. 2012, MNRAS, 426, 120

Fritz, J., Franceschini, A., \& Hatziminaoglou, E. 2006, MNRAS, 366, 767

Furlong, M., Bower, R. G., Theuns, T., et al. 2014 [arXiv: 1410. 3485]

Gavazzi, G., Pierini, D., \& Boselli, A. 1996, A\&A, 312, 397
Gawiser, E., Francke, H., Lai, K., et al. 2007, ApJ, 671, 278

Georgakakis, A., Nandra, K., Yan, R., et al. 2008, MNRAS, 385, 2049

Georgakakis, A., Coil, A. L., Willmer, C. N. A., et al. 2011, MNRAS, 418, 2590

Georgakakis, A., Pérez-González, P. G., Fanidakis, N., et al. 2014, MNRAS, 440,339

Giovannoli, E., Buat, V., Noll, S., Burgarella, D., \& Magnelli, B. 2011, A\&A, 525, A 150

Gladders, M. D., Oemler, A., Dressler, A., et al. 2013, ApJ, 770, 64

Goto, T. 2006, MNRAS, 369, 1765

Guo, K., Zheng, X. Z., \& Fu, H. 2013, ApJ, 778, 23

Hainline, K. N., Shapley, A. E., Greene, J. E., et al. 2012, ApJ, 760, 74

Harrison, C. M., Alexander, D. M., Mullaney, J. R., \& Swinbank, A. M. 2014, MNRAS, 441, 3306

Hatziminaoglou, E., Fritz, J., Franceschini, A., et al. 2008, MNRAS, 386, 1252

Hatziminaoglou, E., Fritz, J., \& Jarrett, T. H. 2009, MNRAS, 399, 1206

Hatziminaoglou, E., Omont, A., Stevens, J. A., et al. 2010, A\&A, 518, L33

Hayward, C. C., \& Smith, D. J. B. 2015, MNRAS, 446, 1512

Hernán-Caballero, A., Alonso-Herrero, A., Pérez-González, P. G., et al. 2014, MNRAS, 443, 3538

Hopkins, A. M. 2004, ApJ, 615, 209

Hopkins, P. F., Hernquist, L., Cox, T. J., et al. 2006, ApJS, 163, 1

Ilbert, O., Salvato, M., Le Floc'h, E., et al. 2010, ApJ, 709, 644

Ilbert, O., McCracken, H. J., Le Fèvre, O., et al. 2013, A\&A, 556, A55

Kauffmann, G., Heckman, T. M., Tremonti, C., et al. 2003a, MNRAS, 346, 1055

Kauffmann, G., Heckman, T. M., White, S. D. M., et al. 2003b, MNRAS, 341, 33

Kauffmann, G., White, S. D. M., Heckman, T. M., et al. 2004, MNRAS, 353, 713

Kennicutt, Jr., R. C. 1998, ARA\&A, 36, 189

King, A. 2003, ApJ, 596, L27

King, A. 2005, ApJ, 635, L121

Kormendy, J., \& Ho, L. C. 2013, ARA\&A, 51, 511

Lagos, C. D. P., Lacey, C. G., Baugh, C. M., Bower, R. G., \& Benson, A. J. 2011, MNRAS, 416, 1566

Lang, P., Wuyts, S., Somerville, R. S., et al. 2014, ApJ, 788, 11

Lanzuisi, G., de Rosa, A., Ghisellini, G., et al. 2012, MNRAS, 421, 390

Laurent, O., Mirabel, I. F., Charmandaris, V., et al. 2000, A\&A, 359, 887

Lee, S.-K., Idzi, R., Ferguson, H. C., et al. 2009, ApJS, 184, 100

Lee, S.-K., Ferguson, H. C., Somerville, R. S., Wiklind, T., \& Giavalisco, M. 2010, ApJ, 725, 1644

Lee, K.-S., Dey, A., Reddy, N., et al. 2011, ApJ, 733, 99

Lusso, E., Comastri, A., Vignali, C., et al. 2011, A\&A, 534, A110

Lusso, E., Hennawi, J. F., Comastri, A., et al. 2013, ApJ, 777, 86

Magdis, G. E., Daddi, E., Béthermin, M., et al. 2012, ApJ, 760, 6

Maraston, C. 2005, MNRAS, 362, 799

Maraston, C., Pforr, J., Renzini, A., et al. 2010, MNRAS, 407, 830

Marchesini, D., van Dokkum, P. G., Förster Schreiber, N. M., et al. 2009, ApJ, 701, 1765

Michałowski, M. J., Dunlop, J. S., Cirasuolo, M., et al. 2012, A\&A, 541, A85

Mitchell, P. D., Lacey, C. G., Baugh, C. M., \& Cole, S. 2013, MNRAS, 435, 87 
L. Ciesla et al.: Derivation of stellar mass and SFR in AGN host galaxies

Mitchell, P. D., Lacey, C. G., Cole, S., \& Baugh, C. M. 2014, MNRAS, 444, 2637

Mullaney, J. R., Alexander, D. M., Goulding, A. D., \& Hickox, R. C. 2011, MNRAS, 414, 1082

Mullaney, J. R., Pannella, M., Daddi, E., et al. 2012, MNRAS, 419, 95

Muzzin, A., Marchesini, D., Stefanon, M., et al. 2013, ApJ, 777, 18

Nandra, K., Georgakakis, A., Willmer, C. N. A., et al. 2007, ApJ, 660, L11

Nenkova, M., Sirocky, M. M., Ivezić, Ž., \& Elitzur, M. 2008, ApJ, 685, 147

Noeske, K. G., Faber, S. M., Weiner, B. J., et al. 2007a, ApJ, 660, L47

Noeske, K. G., Weiner, B. J., Faber, S. M., et al. 2007b, ApJ, 660, L43

Noll, S., Burgarella, D., Giovannoli, E., et al. 2009, A\&A, 507, 1793

Pacifici, C., Charlot, S., Blaizot, J., \& Brinchmann, J. 2012, MNRAS, 421, 2002

Papovich, C., Dickinson, M., \& Ferguson, H. C. 2001, ApJ, 559, 620

Papovich, C., Finkelstein, S. L., Ferguson, H. C., Lotz, J. M., \& Giavalisco, M. 2011, MNRAS, 412, 1123

Pforr, J., Maraston, C., \& Tonini, C. 2012, MNRAS, 422, 3285

Pozzetti, L., Bolzonella, M., Lamareille, F., et al. 2007, A\&A, 474, 443

Reddy, N. A., Pettini, M., Steidel, C. C., et al. 2012, ApJ, 754, 25

Rodighiero, G., Daddi, E., Baronchelli, I., et al. 2011, ApJ, 739, L40

Rosario, D. J., Santini, P., Lutz, D., et al. 2012, A\&A, 545, A45

Rosario, D. J., Santini, P., Lutz, D., et al. 2013, ApJ, 771, 63

Rovilos, E., Georgantopoulos, I., Akylas, A., et al. 2014, MNRAS, 438, 494

Saez, C., \& Chartas, G. 2011, ApJ, 737, 91

Salim, S., Rich, R. M., Charlot, S., et al. 2007, ApJS, 173, 267

Salim, S., Dickinson, M., Michael Rich, R., et al. 2009, ApJ, 700, 161

Sánchez, S. F., Jahnke, K., Wisotzki, L., et al. 2004, ApJ, 614, 586
Santini, P., Rosario, D. J., Shao, L., et al. 2012, A\&A, 540, A109

Schaerer, D., de Barros, S., \& Sklias, P. 2013, A\&A, 549, A4

Schawinski, K., Virani, S., Simmons, B., et al. 2009, ApJ, 692, L19

Schawinski, K., Urry, C. M., Simmons, B. D., et al. 2014, MNRAS, 440, 889

Silk, J., \& Rees, M. J. 1998, A\&A, 331, L1

Silva, L., Maiolino, R., \& Granato, G. L. 2004, MNRAS, 355, 973

Silverman, J. D., Lamareille, F., Maier, C., et al. 2009, ApJ, 696, 396

Sparre, M., Hayward, C. C., Springel, V., et al. 2015, MNRAS, 447, 3548

Speagle, J. S., Steinhardt, C. L., Capak, P. L., \& Silverman, J. D. 2014, ApJS, 214,15

Springel, V., White, S. D. M., Jenkins, A., et al. 2005, Nature, 435, 629

Strateva, I., Ivezić, Ž., Knapp, G. R., et al. 2001, AJ, 122, 1861

Tacconi, L. J., Neri, R., Genzel, R., et al. 2013, ApJ, 768, 74

Tombesi, F., Cappi, M., Reeves, J. N., et al. 2010, A\&A, 521, A57

Tombesi, F., Cappi, M., Reeves, J. N., \& Braito, V. 2012, MNRAS, 422, L1

Walcher, C. J., Lamareille, F., Vergani, D., et al. 2008, A\&A, 491, 713

Walcher, J., Groves, B., Budavári, T., \& Dale, D. 2011, Ap\&SS, 331, 1

Weedman, D., Charmandaris, V., \& Zezas, A. 2004, ApJ, 600, 106

Wild, V., Heckman, T., \& Charlot, S. 2010, MNRAS, 405, 933

Wu, Y., Charmandaris, V., Huang, J., Spinoglio, L., \& Tommasin, S. 2009, ApJ, 701,658

Wuyts, S., Franx, M., Cox, T. J., et al. 2009, ApJ, 696, 348

Wuyts, S., Förster Schreiber, N. M., van der Wel, A., et al. 2011, ApJ, 742, 96

Xue, Y. Q., Brandt, W. N., Luo, B., et al. 2010, ApJ, 720, 368

Yang, Y. 2005, Biometrika, 92, 937

Zheng, X. Z., Bell, E. F., Papovich, C., et al. 2007, ApJ, 661, L41 